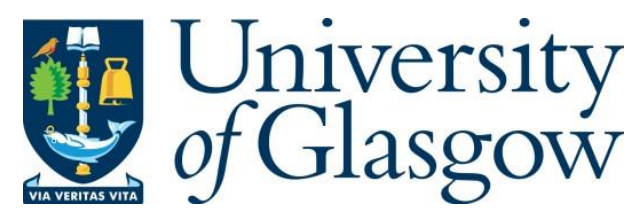

Zhang, Y., Li, D., Qiao, D. and Zhang, L. (2021) Analysis of indoor THz communication systems with finite-bit DACs and ADCs. IEEE Transactions on Vehicular Technology, (doi: 10.1109/TVT.2021.3123380).

There may be differences between this version and the published version. You are advised to consult the publisher's version if you wish to cite from it.

$\underline{\text { http://eprints.gla.ac.uk/257699/ }}$

Deposited on: 25 October 2021

Enlighten - Research publications by members of the University of Glasgow http://eprints.gla.ac.uk 


\title{
Analysis of Indoor THz Communication Systems with Finite-bit DACs and ADCs
}

\author{
Yujiao Zhang, Dan Li, Deli Qiao, and Lei Zhang
}

\begin{abstract}
Terahertz (THz) communication is foreseen to be a key technology for the $6 \mathrm{G}$ wireless communications. In this paper, the performance of downlink Terahertz (THz) communication systems with low-resolution digital-to-analog converters (DACs) at the Access Point (AP) and/or finite-bit analog-to-digital converters (ADCs) at the user sides are investigated. Array-ofsubarrays architecture is assumed at the AP, where each RF chain uniquely activates a disjoint subset of antennas, each of which is connected to an exclusive phase shifter. Hybrid precodings including maximum ratio transmission (MRT) and zero-forcing (ZF) precoding are considered. The best beamsteering direction for the phase shifters in the large subarray antenna regime is first proved to be the direction of the line-of-sight (LOS) path. Subsequently, the closed-form expression of the lower-bound of the achievable rate in the large subarray antenna regime is derived, which is the same for both MRT and ZF and is independent of the transmit power. Moreover, numerical results show that the rate loss caused by the phase uncertainties can be negligible in large subarray antenna regime and moderate resolution DACs and ADCs can achieve performance close to the infinite-resolution one. Numerical results validating the analysis are provided as well. Overall, the impact of finite-bit precoding and finite-bit ADCs on the achievable rate of indoor $\mathrm{THz}$ communication systems is characterized.
\end{abstract}

Index Terms-Terahertz communication systems, array-ofsubarrays, finite-bit DAC, finite-bit ADC, hybrid precoding.

\section{INTRODUCTION}

In the recent years, the exponential increase of mobile traffic and the desirable requirement of higher data rate promote the development of advanced transmission techniques [1]-[3]. It is predicted that more spectral bands will be required in the future wireless communications. Therefore, the Terahertz(THz) band, which is from $0.1 \mathrm{THz}$ to $10 \mathrm{THz}$, has been introduced to be a solution on ultra-high-speed communications. The available bandwidth in the $\mathrm{THz}$ band is one order of magnitude larger than the microwave frequency band, which is from $30 \mathrm{GHz}$ to $300 \mathrm{GHz}$. THz frequency band is large and

This paper has been presented in part at the International Conference on Wireless Communications and Signal Processing (WCSP) 2018 [61] and IEEE Global Communications Conference (Globecom) 2018 [62].

This work is supported in part by the National Natural Science Foundation of China (61671205), in part by Shanghai Rising-Star Program (No. 21QA1402700) and also in part by the open research fund of National Mobile Communications Research Laboratory, Southeast University (No.2020D02).

Y. Zhang is with the School of Communication and Electronic Engineering, East China Normal University, Shanghai, China, 200241. (email:52191214002@stu.ecnu.edu.cn).

D. Li is with Huawei Technologies. (email:51161214012@stu.ecnu.edu.cn).

D. Qiao is with the School of Communication and Electronic Engineering, East China Normal University, Shanghai, China, 200241(e-mail: dlqiao@ce.ecnu.edu.cn).

L. Zhang is with the School of Engineering, University of Glasgow, Glasgow, U.K. G12 8QQ (e-mail: lei.zhang @glasgow.ac.uk). unoccupied, which can be exploited to address the spectrum scarcity and capacity limitation of current communication systems. However, the $\mathrm{THz}$ band suffers from high path loss and molecular absorption. To deal with it, a large scale of antennas are introduced for $\mathrm{THz}$ communications [7]. Thanks to the very short wavelength of $\mathrm{THz}$ signals, antennas can be carried out in a small area.

More attention has paid to modeling and analysis of $\mathrm{THz}$ communications recently (e.g., [8]-[15] and references therein). For instance, in [8], a new propagation model has been developed which includes the molecular absorption in the $\mathrm{THz}$ band. The indoor model has been proposed in [9] and its channel range is from $275 \mathrm{GHz}$ to $325 \mathrm{GHz}$. Considering the different distances from users to access point(AP), the distance-aware multi-carrier transmission has been characterized in [12], by means of utilizing the distance frequency dependent transmission windows in the $\mathrm{THz}$ band. Besides, different situations have been taken into account. The indoor $\mathrm{THz}$ communications with antenna subarrays for one user assuming prefect channel state information (CSI) have been presented in [13], while the similar model for multi-users with partial CSI has been analyzed in [14]. The authors in [15] have characterized that there are promotions on both the spectral and energy efficiency by applying hybrid precoding and array-of-subarray structure. However, it is noteworthy that high-resolution digital-to-analog converters (DACs) and analog-to-digital converters (ADCs) are presumed in the above literatures. These hypotheses can ignore the quantization error, whereas the cost and power consumption may be high.

Therefore, applications of low-resolution DACs and ADCs have aroused interests of the academia, especially for the communication systems with large scale of antenna arrays [21]-[29]. On one hand, many researches in different aspects have been done on quantized precoding for massive MIMO with low-resolution DACs in micro-/millimeter-wave communication systems. For instance, what has been indicated in [21] is that the performance of the MIMO systems equipped with 3-4 bits DACs is on the verge of that in the systems with infinite bits DACs. The authors in [27] have analyzed the energy efficiency of the massive MIMO with low-resolution DACs in mmWave. They have taken hybrid precoding schemes with two different kinds of antenna array structures into consideration. One is the fully-connected structure, which any RF chain drives all antennas with individual phase-shifters, while another one is partially-connected structure, which each $\mathrm{RF}$ chain is only connected to one subarray of antennas with individual phase-shifters. Both have been thought to use AQN model as the quantization error model due to its adaptability of 
low-power regime. In [27], partially-connected structure have gained better energy efficiency. Meanwhile, spectral efficiency cannot be effectively improved by increasing the resolution of DACs.

On the other hand, the low-resolution ADCs have attracted more attention on the analysis of mirco-/millimeter-wave communication systems depending on their low hardware cost and circuit power consumption (e.g., [31]-[41] and references therein). For instance, what has been studied in [33] is the influence on low-resolution ADCs in the uplink massive MIMO communication system. It has indicated the achievement of reliable communication with low-resolution ADCs. The performance on 3-bit resolution of ADCs can be close to that of infinite resolution. An architecture of single-user MIMO system with the hybrid analog/digital precoding architecture and one-bit ADCs is investigated in [37], which showed that designing the hybrid precoding architecture with proposed method can improve the achievable rates effectively. In [41], the authors have derived the closed-form sum rate expressions for a multiuser massive MIMO amplify-and-forward relay uplink in case of both perfect and imperfect CSI with lowresolution ADCs, and showed that the low-resolution ADCs can significantly improve the energy efficiency.

Moreover, the joint effect of finite-bit ADCs at user sides and low-resolution DACs at the base station for mmwave communications has been studied as well in [44]-[48]. For instance, It has been shown in [45] that finite-bit ADCs cause more rate loss than DACs with the same resolution. In [46], a closed-form achievable rate is obtained and a power allocation is proposed to compensate for the rate loss caused by the coarse quantization. A multipair massive MIMO relaying system with mixed-resolution ADCs and DACs at the relay has been investigated in [47], which showed that the mixedADC/DAC architecture can attain a considerable rate and energy efficiency simultaneously. Besides, A specific perspectives of systems with low-resolution ADCs and oversampling at the receiver are considered in [49], which showed that a higher sampling rate can reduce the loss caused by the quantization. But it might not suitable for the $\mathrm{THz}$ communication systems due to the high frequency.

In this paper, we investigate the indoor $\mathrm{THz}$ communications with finite-bit DACs at the AP and finite-bit ADCs at the user sides. We incorporate the distance-aware multicarrier transmission strategy to support multi-user transmissions in the $\mathrm{THz}$ band [12]. We adopt the array-of-subarrays structure, where each RF chain drives only a subarray of antennas with individual phase shifters [15]. We would like to note that antenna and array calibration is essential for the deployment of the array-of-subarrays structure. In [54][58], different approaches have been proposed to combat the deconstructive effect of partially calibrated subarrays. In this work, we assume that the antennas and subarrays have been calibrated or the negative impact can be ignored. We assume the hybrid precoding scheme in which users are first divided into groups based on beam steering angles confined by the $\mathrm{THz}$ channel characteristics in the analog domain. It is also assumed that users in the same group are assigned orthogonal frequencies based on the distance-aware multi-carrier scheme with digital precoding in the baseband. After describing the system input-output relationships, we give the lowerbound of the achievable rate in different sceneries depending on the deployment of finite-bit DACs and finite-bit ADCs. The main objective is to analyze the effect of quantized distortion on transmission performance and the impact of large size antenna subarray for $\mathrm{THz}$ communications. The main contributions and results of this paper are summarized as follows:

- We investigate three different scenarios of the downlink $\mathrm{THz}$ communication systems, including finite-bit DACs only, finite-bit ADCs only and both finite-bit DACs and finite-bit ADCs in the system.

- We consider the large subarray antenna regime, and prove that the analog beamforming angles for each group are decided by the line-of-sight (LOS) path due to the strong directionality of the antenna subarray in the indoor $\mathrm{THz}$ communications.

- We derive the closed-form expressions for the lowerbound on the achievable rate in different scenarios, and characterize the impact of phase uncertainties on the rate degradation in the single-user case. We show that indoor $\mathrm{THz}$ communication systems with low-resolution ADCs and/or DACs should work in low power regime to be power efficient.

- We also provide the numerical results verifying the analysis and note that low-resolution ADCs cause more rate loss compared to low-resolution DACs in the considered $\mathrm{THz}$ communication system. Remarkably, we demonstrate that moderate resolution DACs and ADCs can achieve Tbps for the indoor $\mathrm{THz}$ communication systems.

This paper is organized as follows. In Section II, the system model and preliminaries on indoor $\mathrm{THz}$ channel model and hybrid precoding are discussed. Main results on the analysis in the large subarray antenna regime with low-resolution DACs/ADCs are presented in Section III, with numerical results given in Section IV. Finally, Section V concludes this paper with some lengthy proofs in Appendices.

Notation: $\mathbf{C}^{T}, \mathbf{C}^{H}$, and $\operatorname{tr}(\mathbf{C})$ denote the transpose, Hermitian and trace of matrix $\mathbf{C}$, respectively. $\operatorname{diag}(\mathbf{C})$ denotes the main diagonal of the matrix $\mathbf{C}$, while $\operatorname{diag}(\mathbf{c})$ represents the diagonal matrix generated by the vector c. $\Re\{\cdot\}$ and $\Im\{\cdot\}$ are the real and imaginary parts of complex values, respectively. $[\mathbf{C}]_{\iota, \ell}$ represents the entry on the $\iota$-th row and the $\ell$-th column. $\mathbb{E}[\cdot]$ is the expectation, $\delta(\cdot)$ is the Dirac delta function and $\Phi(x)=\frac{1}{2 \pi} \int_{-\infty}^{x} e^{-u^{2} / 2} d u$ is the cumulative distribution function of the standard normal distribution.

\section{System Model AND PRELIMINARIES}

In this section, we will first briefly introduce the system model, and then the indoor $\mathrm{THz}$ channel model and describe the models of low-resolution DACs/ADCs.

\section{A. System Model}

We consider the downlink $\mathrm{THz}$ communication systems with finite-bit DACs at the transmitter and/or finite-bit ADCs at user side as illustrated in Fig.1. In [16]-[18], different methods are used to estimate the channel on high frequency 


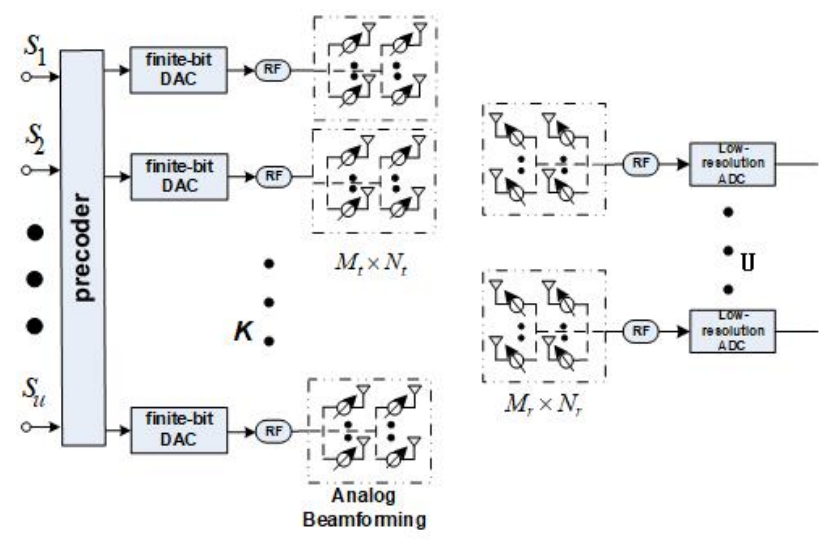

Fig. 1. The indoor $\mathrm{THz}$ communication system model with finite-bit DACs and finite-bit ADCs. Typical components such as demodulator, channel estimator, and sub-band allocator are not included in this illustration to emphasize the DAC/ADC components.

band and the results are acceptable. To reduce the complexity of the problem, we assume that the channel is perfectly estimated, so the performance limitation can be determined by this hypothesis. We presume the array-of-subarrays architecture, which is one kind of hybrid precoding structure and has been shown to have better performance on both the spectral and energy efficiency in the Terahertz band with reduced complexity [14]. Especially, the AP is equipped with $K$ antenna subarrays. Each subarray is equipped with $M_{t} \times N_{t}$ tightly-packed antenna elements, each of which is driven by an analog phase shifter. The AP is supposed to serve $U$ users with different distances. One user is assigned only one subarray of size $M_{r} \times N_{r}$. In general, $K$ is presupposed to be greater than $U$ in order to provide sufficient degrees of freedom to all users.

1) Finite-bit DACs only. Each subarray at the AP is connected to one RF chain and a finite-bit DAC while the users are equipped with infinite-bit ADCs, i.e., the low-resolution ADCs are replaced by infinite-bit ADCs in Fig.1.

2) Finite-bit ADCs only. Each subarray at the user sides is connected to one RF chain with finite-bit ADC while infinite-bit DACs for the AP, i.e., the finite-bit DACs are replaced by infinite-bit DACs in Fig.1.

3) Both finite-bit DACs and finite-bit ADCs. Each subarray of the AP and the users is equipped with finite-bit DACs and finite-bit ADCs, respectively, i.e., the system model in Fig.1.

The subarray antenna element spacing $a$ is normally set to be less than the wavelength while the spacing between the adjacent subarrays is much larger than the wavelength. Based on this, independent channels can be expected for different subarrays. Besides, the distance from any user to the AP is presumed to be much larger than the spacing between the subarrays such that the distance between user $i$ and any subarray is the same and indicated as $d_{i}$.

\section{B. Indoor Terahertz Channel Model}

Following the ray tracing method, the wideband channel response can be represented as the sum of the contributions of the individual subbands [10], [11], where the channel response for each subband can be measured accordingly. Assume each subband to be within the coherence bandwidth such that the channel experiences flat frequency response in each subband. As will be discussed later, multi-carrier transmission scheme is assumed and the transmission bandwidth for each user is composed of multiple subbands. The multi-ray channel response for antenna subarray $k$ of a subband can be written as [11], [13]

$$
\begin{aligned}
& \mathbf{F}_{\text {sub }_{k}}(f, d) \\
= & \sqrt{M_{t} N_{t} M_{r} N_{r}}\left[\eta_{L}(f, d) \Omega_{t} \Omega_{r} \mathbf{a}_{r}\left(\psi_{L}^{r}, \phi_{L}^{r}\right) \mathbf{a}_{t}^{H}\left(\psi_{L}^{t}, \phi_{L}^{t}\right)\right. \\
+ & \left.\sum_{i=1}^{J} \eta_{i}(f, d) \Omega_{t} \Omega_{r} \mathbf{a}_{r}\left(\psi_{i}^{r}, \phi_{i}^{r}\right) \mathbf{a}_{t}^{H}\left(\psi_{i}^{t}, \phi_{i}^{t}\right)\right],
\end{aligned}
$$

where $M_{t}, N_{t}$ and $M_{r}, N_{r}$ are the antenna sizes of transmitter and receiver, separately. $\Omega_{t}$ and $\Omega_{r}$ are the transmit and receive antenna gains. $\mathbf{a}_{t}$ and $\mathbf{a}_{r}$ are the array steering vectors which are explained in detail below. $J$ is represented for the number of non-line-of-sight (NLOS) rays. The channel coefficient $\eta_{i}(f, d)=\left|\eta_{i}(f, d)\right| e^{j \vartheta_{i}}$ is for the ray $i$ where $\vartheta_{i}$ is the independent phase shift of the path $i$. Meanwhile, $\psi_{i}^{t}, \phi_{i}^{t}$ and $\psi_{i}^{r}, \phi_{i}^{r}$ are the corresponding azimuth and elevation angles of departure and arrival (AoD/AoA) for path $i$, respectively. Generally, relatively large antenna gains with directional antenna elements are required to oppose the high path loss in $\mathrm{THz}$ bands [12, Fig. 2].

For an $(M, N)$-element uniform planar array, the array steering vector is given by

$$
\begin{aligned}
\mathbf{a}(\psi, \phi)= & \frac{1}{\sqrt{M N}}\left[1, \cdots, e^{j \frac{2 \pi a}{\lambda}[m \cos \psi \sin \phi+n \sin \psi \sin \phi]},\right. \\
& \left.\cdots, e^{j \frac{2 \pi a}{\lambda}[(M-1) \cos \psi \sin \phi+(N-1) \sin \psi \sin \phi]}\right]^{T},
\end{aligned}
$$

where $m$ and $n$ are the antenna element indexes with $0 \leq m \leq$ $M-1,0 \leq n \leq N-1, \lambda$ is the wavelength and $a$ is the antenna element spacing. We would like to note that $d$ here is only adopted as an indication of the user with distance $d$ from the AP. For different propagation paths, the coefficients $\left\{\eta_{i}(f, d)\right\}$ will be calculated considering the path lengths correspondingly [11, (18)].

The $\mathrm{THz}$ signals are easily affected by the atmospheric attenuation and molecular absorption. The path gain of the LOS path is expressed as [8]

$$
\left|\eta_{L}(f, d)\right|^{2}=\mathfrak{L}_{a s p}(f, d)=\left(\frac{c}{4 \pi f d}\right)^{2} e^{-\kappa_{a b s}(f) d},
$$

where $c$ is the speed of light in free space, $f$ is the carrier frequency, $d$ is the path length, and $\kappa_{a b s}$ is the frequencydependent medium absorption coefficient, which is decided by the transmission medium at a molecular level. On the other hand, on account of the sub-millimeter wavelength of the $\mathrm{THz}$ signals, the indoor surfaces are rough at $\mathrm{THz}$ frequency, which will put additional loss into the NLOS rays [9]. Therefore, the path gain of the $i$-th NLOS ray with one reflection satisfies

$$
\left|\eta_{i}(f, d)\right|^{2}=\Gamma_{i}^{2}(f) \mathfrak{L}_{a s p}(f, d),
$$

where $\Gamma$ is the reflection coefficient composed of the Fresnel 
reflection coefficient and the Rayleigh roughness factor. It is noteworthy that we only consider two reflections in the $\mathrm{THz}$ band because of its high reflection loss [9], and the associated path gain can be written similarly to (4). Therefore, the $\mathrm{THz}$ channel is deemed to have limited number of paths in the case. For interested readers, please refer to [8]-[11] and references therein for detailed descriptions of the $\mathrm{THz}$ channel modeling.

\section{Transmission Windows}

In the $\mathrm{THz}$ communication systems, the molecular absorption will influence the propagation severely in certain frequencies. Then, the frequency band will be split into different transmission windows, which band are related to communication distances. These kind of windows play an important role on designing the transmission strategies in $\mathrm{THz}$ band [12]. For instance, distance-aware multi-carrier transmission has been proposed and analyzed for indoor $\mathrm{THz}$ communications, where the transmission windows, $0.6-0.7 \mathrm{THz}$ and $0.8-0.95 \mathrm{THz}$, will be assigned to the user with $10 \mathrm{~m}$ distance to the AP, while the transmission windows, $0.5-0.6 \mathrm{THz}, 0.7-0.8 \mathrm{THz}$, and $0.95-1$ $\mathrm{THz}$, will be allocated to the user $1 \mathrm{~m}$ away. In this paper, we adopt such distance-aware multi-carrier transmission, and denote the subcarrier index as $w$ with central frequency $f^{w}$ and bandwidth $B$ and $\mathcal{W}_{u}$ as the set of subcarrier index for user $u$. We would like to note that the channel experienced for each subcarrier of one subarray is assumed to be flat and modeled as (1). This is different from the micro/mm-wave communication systems, where the whole bandwidth can be utilized for any user's data transmissions.

\section{Hybrid Beamforming}

We consider hybrid beamforming strategy, in which digital beamforming at baseband and analog beamforming at each antenna subarray are performed. In this scheme, the users are first divided into user groups based on the transmit analog beamforming angles, and then after obtaining the effective channel information of the subarrays with the analog beamforming, digital precoding such as maximal ratio transmission (MRT) and zero-forcing (ZF) are applied. We would like to note that unlike the micro/mm-wave communication systems, different sets of antenna subarrays are assigned to different groups of users. In case that the users are located closely with similar LOS directions, user scheduling can be taken advantage of to avoid simultaneous transmissions to such users. It will be interesting to design the optimal user scheduling policy subject to different constraints, such as minimum rate constraints [60], and are left for future study.

1) Analog Beamforming: Note that the $\mathrm{THz}$ phase shifter$\mathrm{s}$ are designed to be implemented by digitally controlled graphene integrated gates in [52], where only finitely quantized angles are available, i.e., the analog beamforming angles can only take a few angles. Thus, we can only select the analog beamforming angles from the finite-size beamsteering codebooks in $2 \pi$ as in [53]. Specifically, subarrays activate a set of antennas to produce a beam pre-scanning with an angular separation $\varphi$, the different users in the same angle section are viewed as one group. Moreover, we assume that users in the same group are assigned orthogonal frequencies according to the distance-aware multi-carrier transmission scheme [14].

Denote the user set in group $q$ as $\mathcal{U}_{q}$. The users in the same group share the set of antenna subarrays $\mathcal{K}_{q}$, where $K=$ $\sum_{q \in Q} \mathcal{K}_{q}$ with $Q$ representing the number of groups.

The choice of the transmit and receive beamforming angles are obtained based on maximizing the weighted sum of the channel gain of the equivalent channel. Specifically, the transmit beamforming angles for the users in group $q,\left(\tilde{\psi}_{g}^{t}, \tilde{\phi}_{q}^{t}\right)$, and receive beamforming angles for user $u$ in group $q,\left(\tilde{\psi}_{u, q}^{r}, \tilde{\phi}_{u, q}^{r}\right)$, can be selected from the codebook as follows

$$
\begin{aligned}
& \left\{\left(\tilde{\psi}_{q}^{t}, \tilde{\phi}_{q}^{t}\right),\left(\tilde{\psi}_{u, q}^{r}, \tilde{\phi}_{u, q}^{r}\right)\right\} \\
= & \arg \max \underset{\substack{\left(\psi_{q}^{t}, \phi_{k, q}^{t}\right) \in \Theta^{t},\left(\psi_{u, q}^{t}, \phi_{u, q}^{r}\right) \in \Theta^{r}}}{\sum_{u \in \mathcal{U}_{q}}} \sum_{w \in \mathcal{W}_{u}} \frac{\left\|\mathbf{h}\left(f_{u}^{w}, d_{u}\right)\right\|^{2}}{\left|\eta_{L}\left(f_{u}^{w}, d_{u}\right)\right|^{2}}, \forall q=1 \ldots Q,
\end{aligned}
$$

where $\mathbf{h}\left(f_{u}^{w}, d_{u}\right)=\left[h_{1}\left(f_{u}^{w}, d_{u}\right), \ldots, h_{k}\left(f_{u}^{w}, d_{u}\right), \ldots, h_{\mathcal{K}_{q}}\left(f_{u}^{w}, d_{u}\right)\right] \in$ $\mathbb{C}^{1 \times K_{q}}$ is the effective channel between the AP and user $u$ with element $h_{k}\left(f_{u}^{w}, d_{u}\right)$ denoting the effective channel between the user $u$ and the $k$-th subarray of the AP for $k \in \mathcal{K}_{q}$ given by

$$
h_{k}\left(f_{u}^{w}, d_{u}\right)=\mathbf{a}_{r}^{H}\left(\psi^{r}, \phi^{r}\right) \mathbf{F}_{s u b_{k}}\left(f_{u}^{w}, d_{u}\right) \mathbf{a}_{t}\left(\psi^{t}, \phi^{t}\right),
$$

where $d_{u}$ is the distance from the AP to user $u$. $\Theta^{t}$ and $\Theta^{r}$ denote the transmit and receive beamforming codebook, respectively. $\Theta^{t}$ and $\Theta^{r}$ are composed of $\left(\psi^{t}, \phi^{t}\right)$ and $\left(\psi^{r}, \phi^{r}\right)$, satisfying $\sin \left(\psi^{t}\right)=-1+\frac{2 m-1}{M_{t}}, m=1, \ldots, M_{t}$, $\sin \left(\phi^{t}\right)=-1+\frac{2 n-1}{N_{t}}, n=1, \ldots, N_{t},\left(\psi^{r}, \phi^{r}\right) \sin \left(\psi^{r}\right)=$ $-1+\frac{2 m-1}{M_{r}}, m=1, \ldots, M_{r}$, and $\sin \left(\phi^{r}\right)=-1+\frac{2 n-1}{N_{r}}, n=$ $1, \ldots, N_{r}$, respectively. Normalization based on the LOS path gain here is to ensure the fairness among users. Otherwise, the short-distance user will dominate the choice due to the large path loss for long-distance users in $\mathrm{THz}$ band. Note that there will be one transmit beamforming angle and $K_{q}$ receive beamforming angles obtained for each group, and hence in total $Q$ transmit beamforming angles and $\sum_{q=1}^{Q} K_{q}$ receive beamforming angles obtained.

Denote $\tilde{\mathbf{h}}\left(f_{u}^{w}, d_{u}\right)$ as the effective channel of one antenna subarray for user $u$ in group $q$ with analog beamforming angles obtained from (5). According to the distance-aware multi-carrier transmission strategy, there might be different numbers of users in subcarrier $w$. Denote $\mathcal{U}^{w}$ as the set of users sharing the same carrier with central frequency $f_{u}^{w}$ with total number $U^{w}$. Let $q_{u}$ be the group index associated with the users $u \in \mathcal{U}^{w}$. Then, we know that $\mathcal{K}^{w}$ represents the set of subarrays transmitting signals in subcarrier $w$ with total number $K^{w}=\sum_{u \in \mathcal{U}^{w}} K_{q_{u}}$. We assume $K^{w} \geq U^{w}$ to guarantee simultaneous transmissions of $U^{w}$ streams.

Let $\mathbf{F}_{u}=\left[\mathbf{F}_{1, u}, \mathbf{F}_{2, u}, \ldots, \mathbf{F}_{K^{w}, u}\right] \in \mathbb{C}^{M_{r} N_{r} \times K^{w} M_{t} N_{t}}$ be the channel matrix for user $u \in \mathcal{U}^{w}$ with respect to the subarrays in $\mathcal{K}^{w}$. Then, the effective channel vector $\tilde{\mathbf{h}}_{u}\left(f_{u}^{w}, d_{u}\right) \in \mathbb{C}^{1 \times K^{w}}$ for user $u$ in group $q$ with analog beamforming is given by

$$
\tilde{\mathbf{h}}_{u}\left(f_{u}^{w}, d_{u}\right)=\mathbf{v}_{u}^{H} \mathbf{F}_{u} \mathbf{D},
$$

where $\mathbf{v}_{u}=\mathbf{a}_{r}\left(\tilde{\psi}_{u, q}^{r}, \tilde{\phi}_{u, q}^{r}\right) \in \mathbb{C}^{M_{r} N_{r} \times 1}$ represents the receive analog beamforming vector, and $\mathbf{D} \in \mathbb{C}^{K^{w} M_{t} N_{t} \times K^{w}}$ 
stands for the transmit analog beamforming operation and is a block matrix with diagonal components given by $\left[\mathbf{a}_{t}\left(\tilde{\psi}_{1}^{t}, \tilde{\phi}_{1}^{t}\right), \ldots, \mathbf{a}_{t}\left(\tilde{\psi}_{K^{w}}^{t}, \tilde{\phi}_{K^{w}}^{t}\right)\right]$ corresponding to the transmit beam steering vectors for subarrays $k \in \mathcal{K}^{w}$ and other components all being zero matrix of size $M_{t} N_{t} \times 1$. Specifically,

$$
\mathbf{D}=\left(\begin{array}{cccc}
\mathbf{a}_{t}\left(\tilde{\psi}_{1}^{t}, \tilde{\phi}_{1}^{t}\right) & 0 & \ldots & 0 \\
0 & \mathbf{a}_{t}\left(\tilde{\psi}_{2}^{t}, \tilde{\phi}_{2}^{t}\right) & \ldots & 0 \\
\vdots & \vdots & \vdots & \vdots \\
0 & 0 & \ldots & \mathbf{a}_{t}\left(\tilde{\psi}_{K^{w}}^{t}, \tilde{\phi}_{K^{w}}^{t}\right)
\end{array}\right)
$$

2) Precoding: We would like to note that the instantaneous channel matrix of each user $\mathbf{F}_{u}$ is not needed for the downlink transmissions. Instead, we assume that $\tilde{\mathbf{h}}_{u}\left(f_{u}^{w}, d_{u}\right)$ can be perfectly estimated at the user side and fed back to the AP. The equivalent channel matrix for all users, $\tilde{\mathbf{H}} \in \mathbb{C}^{U^{w} \times K^{w}}$, can be expressed as

$$
\tilde{\mathbf{H}}=\left[\tilde{\mathbf{h}}_{1}^{T}, \ldots, \tilde{\mathbf{h}}_{U^{w}}^{T}\right]^{T} .
$$

Then, maximal ratio transmission (MRT) precoding matrix, $\mathbf{Q}_{M R T} \in \mathbb{C}^{K^{w} \times U^{w}}$, is given by

$$
\mathbf{Q}_{M R T}^{w}=\rho_{M R T} \tilde{\mathbf{H}}^{\mathbf{H}},
$$

where $\rho_{M R T}=\sqrt{P^{w}} / \sqrt{\operatorname{tr}\left(\tilde{\mathbf{H}} \tilde{\mathbf{H}}^{\mathbf{H}}\right)}$.

Zero-forcing $(\mathrm{ZF})$ precoding matrix, $\mathbf{Q}_{Z F} \in \mathbb{C}^{K^{w} \times U^{w}}$, is given by

$$
\mathbf{Q}_{Z F}=\rho_{Z F} \tilde{\mathbf{H}}^{H}\left(\tilde{\mathbf{H}} \tilde{\mathbf{H}}^{H}\right)^{-1}
$$

where $\rho_{Z F}=\sqrt{P^{w}} / \sqrt{\operatorname{tr}\left(\left(\tilde{\mathbf{H}} \tilde{\mathbf{H}}^{H}\right)^{-1}\right)}$.

\section{E. Operations of DACs}

At the baseband, we consider quantized precoding by incorporating the finite-bit DACs at the transmitter side. The transmitted signal can be expressed as

$$
\begin{aligned}
\mathbf{x} & =\mathrm{L}(\mathbf{Q} \mathbf{s})=\sum_{i=0}^{L_{D A C}-1} \ell_{i} \mathbb{1}_{\left[\tau_{i}, \tau_{i+1}\right)} \Re\{\mathbf{Q} \mathbf{s}\} \\
& +j \sum_{i=0}^{L_{D A C}-1} \ell_{i} \mathbb{1}_{\left[\tau_{i}, \tau_{i+1}\right)} \Im\{\mathbf{Q} \mathbf{s}\},
\end{aligned}
$$

where $\mathbf{x} \in \mathbb{C}^{U^{w} \times 1}$ is the precoded transmit signal, $\mathbf{s} \in \mathbb{C}^{U^{w} \times 1}$ is the Gaussian source data with $\mathbb{E}\left\{\mathbf{s s}^{H}\right\}=\mathbf{I}_{U^{w}}, \mathbf{E}(\cdot)$ represents the quantization-mapping function, $\mathbf{Q} \in \mathbb{C}^{K^{w} \times U^{w}}$ denotes the linear precoding operation performed at the baseband with $\operatorname{tr}\left\{\mathbf{Q} \mathbf{Q}^{H}\right\}=P^{w} \cdot L_{D A C}=2^{b_{D A C}}$ and $b_{D A C}$ are the number of DAC levels and DAC bits. For simplicity, DACs are modeled as symmetric uniform quantizers, where $\Delta_{D A C}$ is the step size. The quantization thresholds are $\tau_{i}=\Delta_{D A C}\left(i-\frac{L_{D A C}}{2}\right)$ for $i=1,2, \ldots, L_{D A C}-1$ and $\tau_{0}=-\infty, \tau_{L_{D A C}}=\infty$. The quantization labels are $\ell_{i}=\alpha_{D A C} \Delta_{D A C}\left(i-\frac{L_{D A C}}{2}+\frac{1}{2}\right)$ for $i=0,1, \ldots, L_{D A C}-1$. The choice of the step size determines the distortion of DACs, and $\Delta_{D A C}=2 \dot{A}_{c} / L_{D A C}$, where $\dot{A}_{c}$ is the clipping level of the uniform quantizer.
Moreover, according to the Bussgang theorem [51], we can model the precoding operation as [21][23]

$$
\mathbf{x}=\mathrm{七}(\mathbf{Q s})=\dot{\mathbf{G}} \mathbf{Q} \mathbf{s}+\mathbf{d},
$$

where $\mathbf{d} \in \mathbb{C}^{K^{w} \times 1}$ stands for the distortion introduced by the DACs, and $\dot{\mathbf{G}} \in \mathbb{C}^{K^{w} \times K^{w}}$ is the diagonal matrix modeling the operation of the finite-bit DACs quantization, which is given by

$$
\begin{aligned}
\dot{\mathbf{G}} & =\frac{\alpha_{D A C} \Delta_{D A C}}{\sqrt{\pi}} \operatorname{diag}\left(\mathbf{Q} \mathbf{Q}^{H}\right)^{-\frac{1}{2}} \\
& \times \sum_{i=1}^{L_{D A C}-1} \exp \left[-\Delta_{D A C}^{2}\left(i-\frac{L_{D A C}}{2}\right)^{2} \operatorname{diag}\left(\mathbf{Q} \mathbf{Q}^{H}\right)^{-1}\right]
\end{aligned}
$$

where $\alpha_{D A C}$ is a constant to ensure the power constraint $\|\mathbf{x}\|^{2} \leq P^{w}$

Specifically, when $b_{D A C}=1$, (14) can be simplified as

$$
\dot{\mathbf{G}}_{b_{1}}=\sqrt{\frac{2 P^{w}}{\pi K^{w}}} \operatorname{diag}\left(\mathbf{Q} \mathbf{Q}^{H}\right)^{-\frac{1}{2}},
$$

where $\alpha_{D A C}^{b_{1}}=\sqrt{2 P^{w} /\left(\Delta_{D A C}^{2} K^{w}\right)}$ to satisfy the power constraint is applied.

Then, the system output can be described as

$$
\dot{\mathbf{y}}=\tilde{\mathbf{H}} \mathbf{x}+\mathbf{n}
$$

where $\mathbf{n} \in \mathbb{C}^{U^{w} \times 1}$ is the additive white Gaussian noise (AWGN) vector.

\section{F. Operations of ADCs}

When we only consider the case of the indoor $\mathrm{THz}$ communication systems with finite-bit ADCs at user side, the received vector $\ddot{\mathbf{r}} \in \mathbb{C}^{U^{w} \times 1}$ is given by

$$
\ddot{\mathbf{r}}=\tilde{\mathbf{H Q s}}+\mathbf{n},
$$

where $\mathbf{s} \in \mathbb{C}^{U^{w} \times 1}$ is the Gaussian source data, $\tilde{\mathbf{H}} \in \mathbb{C}^{U^{w} \times K^{w}}$ is the THz channel with analog beamforming. $\mathbf{n} \in \mathbb{C}^{U^{w} \times 1}$ is the AWGN vector.

We consider the optimal non-uniform ADCs since it provides a tractable and effective way of well characterizing the quantization performance [33]. After the ADC quantization and according to the Bussgang theorem [51], the system output can be written as

$$
\ddot{\mathbf{y}}=\ddot{\mathbf{G}} \ddot{\mathbf{r}}+\varpi
$$

where $\varpi \in \mathbb{C}^{U^{w} \times 1}$ stands for the distortion introduced by the ADCs, and $\ddot{\mathbf{G}} \in \mathbb{C}^{U^{w} \times U^{w}}$ is the diagonal matrix modeling the operation of the ADC quantization, which is given by [44]

$$
\ddot{\mathbf{G}}=\left(1-e_{q}\right) \mathbf{I}_{K^{w}},
$$

and the distortion can be written as

$$
\mathbb{E}\left\{\varpi \varpi^{H}\right\}=e_{q}\left(1-e_{q}\right) \mathbb{E}\left\{\operatorname{diag}\left(\dddot{\mathbf{r}}^{H}\right)\right\} .
$$

Specifically, $e_{q}$ is defined as $e_{q}=\frac{E\left\{\|\ddot{\mathbf{y}}-\ddot{\mathbf{r}}\|^{2}\right\}}{E\left\{\|\ddot{\mathbf{y}}\|^{2}\right\}}$, and exemplified as $\{0.3634,0.1175,0.03454$,

$0.009497,0.002499,0.0006642,0.0001660,0.00004151\}$ with 
$b_{A D C}=\{1,2,3,4,5,6,7,8\}$ [43]. Also, considering the case of moderate to high-resolution quantizations, we have the approximation of $e_{q}$ as [34]

$$
e_{q} \approx \frac{\pi \sqrt{3}}{2} 2^{-2 b_{A D C}},
$$

where $b_{A D C}$ specifies the resolution of ADC.

\section{PERFORMANCE ANALYSIS}

In this section, we investigate the achievable rate of the indoor $\mathrm{THz}$ communications with finite-bit DACs and finitebit ADCs in the large subarray antenna regime, in which the number of antennas in each subarray goes to infinity.

\section{A. Finite-bit DACs Only}

We first consider the indoor $\mathrm{THz}$ communication system with finite-bit DACs at the AP. Incorporating (7)-(16) and denoting $\mathbf{q}_{i}$ as the $i$-th column of the precoding matrix $\mathbf{Q}$, the received signal $\dot{y}_{u}$ after receive analog beamforming at user $u$ in group $q$ in subcarrier $w$ can be described as

$$
\begin{aligned}
\dot{y}_{u} & =\tilde{\mathbf{h}}_{u}\left(f_{u}^{w}, d_{u}\right) \dot{\mathbf{G}} \mathbf{q}_{u} s_{u}+\sum_{u^{\prime} \in \mathcal{U}^{w}, u^{\prime} \neq u} \tilde{\mathbf{h}}_{u}\left(f_{u}^{w}, d_{u}\right) \dot{\mathbf{G}} \mathbf{q}_{u^{\prime}} s_{u^{\prime}} \\
& +\tilde{\mathbf{h}}_{u}\left(f_{u}^{w}, d_{u}\right) \mathbf{d}+n_{u},
\end{aligned}
$$

where $\mathbf{q}_{u} \in \mathbb{C}^{K^{w} \times 1}$ denotes the digital precoding vector corresponding to user $u$, and $n_{u}$ is the AWGN with power $N_{0}$.

Note that when characterizing the information rate, the nonlinearity introduced by the DACs prevents one to obtain the closed-form expression for the achievable rate. Fortunately, using the "auxiliary-channel lower bound" proposed in [59], we can obtain the lowerbound of the achievable rate as [21]

$$
\begin{aligned}
& \dot{R}_{u} \geq \mathbb{E}\left[\sum_{w \in \mathcal{W}_{u}} B \log _{2}(1+\right. \\
& \left.\left.\frac{\left|\tilde{\mathbf{h}}_{u}\left(f_{u}^{w}, d_{u}\right) \dot{\mathbf{G}} \mathbf{q}_{u}\right|^{2}}{\sum_{u \neq i}\left|\tilde{\mathbf{h}}_{u}\left(f_{u}^{w}, d_{u}\right) \dot{\mathbf{G}} \mathbf{q}_{i}\right|^{2}+\tilde{\mathbf{h}}_{u}\left(f_{u}^{w}, d_{u}\right) \mathbf{C}_{d d} \tilde{\mathbf{h}}_{u}^{H}\left(f_{u}^{w}, d_{u}\right)+N_{0}}\right)\right],
\end{aligned}
$$

where $\mathbf{C}_{d d}=\mathbb{E}\left[\mathbf{d d}^{H}\right]$ stands for the covariance of the distortion $\mathbf{d}$. This lowerbound provides a good approximation of the achievable rate [59]. Hence, by using (13), $\mathbf{C}_{d d}$ can be expressed as

$$
\mathbf{C}_{d d}=\mathbb{E}\left[\mathbf{d d}^{H}\right]=\mathbb{E}\left[\mathbf{x x}^{H}\right]-\dot{\mathbf{G}} \mathbf{Q} \mathbf{Q}^{H} \dot{\mathbf{G}}=\mathbf{C}_{x x}-\dot{\mathbf{G}} \mathbf{Q} \mathbf{Q}^{H} \dot{\mathbf{G}} .
$$

For one-bit DACs, the closed form of $\mathbf{C}_{d d}$ is given by [23], [50]

$$
\begin{aligned}
\mathbf{C}_{d d}^{b_{1}} & =\frac{2 P^{w}}{\pi K^{w}}\left\{\arcsin \left(\operatorname{diag}\left(\mathbf{Q} \mathbf{Q}^{H}\right)^{-\frac{1}{2}} \Re\left\{\mathbf{Q} \mathbf{Q}^{H}\right\} \operatorname{diag}\left(\mathbf{Q} \mathbf{Q}^{H}\right)^{-\frac{1}{2}}\right)\right. \\
& \left.+j \arcsin \left(\operatorname{diag}\left(\mathbf{Q} \mathbf{Q}^{H}\right)^{-\frac{1}{2}} \Im\left\{\mathbf{Q} \mathbf{Q}^{H}\right\} \operatorname{diag}\left(\mathbf{Q} \mathbf{Q}^{H}\right)^{-\frac{1}{2}}\right)\right\} \\
& -\dot{\mathbf{G}}_{b_{1}} \mathbf{Q} \mathbf{Q}^{H} \dot{\mathbf{G}}_{b_{1}}^{H} .
\end{aligned}
$$

When considering the case of $L_{D A C}>2$, it is hard to have a closed-form expression for $\mathbf{C}_{d d}$ and a numerical method was adopted in [30]. In this paper, to obtain the closed-form expressions for the achievable rate, the diagonal approximation
$\mathbf{C}_{d d}^{\text {diag }}[22]$ is applied and

$$
\mathbf{C}_{d d}^{\text {diag }}=\operatorname{diag}\left(\mathbf{C}_{x x}\right)-\dot{\mathbf{G}} \operatorname{diag}\left(\mathbf{Q} \mathbf{Q}^{H}\right) \dot{\mathbf{G}}^{H} .
$$

Note that the diagonal approximation method can be accurate for moderate to high DAC resolutions [22]. Then, considering (12), $\operatorname{diag}\left(\mathbf{C}_{x x}\right)$ can be written as

$$
\begin{aligned}
\operatorname{diag}\left(\mathbf{C}_{x x}\right) & =2 \sum_{i=0}^{L_{D A C}-1} \ell_{i}^{2} \mathbb{P}\left[\Re\{\mathbf{x}\}=\ell_{i}\right] \\
& =2 \sum_{i=0}^{L_{D A C}-1} \ell_{i}^{2} \mathbb{P}\left[\tau_{i} \leq \Re\{\mathbf{Q} \mathbf{s}\}<\tau_{i+1}\right] \\
& =2 \sum_{i=0}^{L_{D A C}-1} \ell_{i}^{2}\left[\Phi\left(\sqrt{2} \tau_{i+1} \operatorname{diag}\left(\mathbf{Q} \mathbf{Q}^{H}\right)^{-\frac{1}{2}}\right)\right. \\
& \left.-\Phi\left(\sqrt{2} \tau_{i} \operatorname{diag}\left(\mathbf{Q} \mathbf{Q}^{H}\right)^{-\frac{1}{2}}\right)\right] .
\end{aligned}
$$

Incorporating the lower-bound as the achievable rate for each user, the sum rate of the indoor $\mathrm{THz}$ communication systems with finite-bit DACs is then given by

$$
\dot{R}=\sum_{u=1}^{U} \dot{R}_{u} .
$$

Due to the high reflection loss, the power of the first-order reflected path is attenuated by more than $10 \mathrm{~dB}$ on average and the second-order reflection by more than $20 \mathrm{~dB}$ compared with the path gain of LOS path in the THz propagation [9], i.e., $\left|\eta_{L}\right|>\left|\eta_{i}\right|, \forall i$. High directionality in the THz band can be expected, we first have the following result.

Proposition 1: When the number of antennas in each subarray goes to infinity, the transmit and receive beamforming angles for user $u$ in group $q$ are given by

$$
\left\{\left(\tilde{\psi}_{q}^{t}, \tilde{\phi}_{q}^{t}\right),\left(\tilde{\psi}_{u, q}^{r}, \tilde{\phi}_{u, q}^{r}\right)\right\}=\left\{\left(\psi_{u, L}^{t}, \phi_{u, L}^{t}\right),\left(\psi_{u, L}^{r}, \phi_{u, L}^{r}\right)\right\} .
$$

Proof: See Appendix A for details.

Remark 1: From Proposition 1, one simple and effective way to determine the beamforming angles in the analog beamforming domain is to select the AoD/AoA of the LoS path when the number of antennas in each subarray goes to infinity. This can simplify the beamforming design for indoor $\mathrm{THz}$ communication systems.

Henceforth, we assume that the analog beamforming angles are determined through Proposition 1. As a result, when the number of antennas in the subarray goes to infinity, asymptotical orthogonality in analog beamforming can be expected, and (7) can be rewritten as

$\tilde{\mathbf{h}}_{u}\left(f_{u}^{w}, d_{u}\right) \stackrel{M_{t}, N_{t}, M_{r}, N_{r} \rightarrow \infty}{\longrightarrow} \sqrt{M_{t} N_{t} M_{r} N_{r}} \Omega_{t} \Omega_{r} \mathbf{t}_{u}\left(f_{u}^{w}, d_{u}\right)$,

where

$\mathbf{t}_{u}\left(f_{u}^{w}, d_{u}\right)=\left[\mathbf{0}_{1 \times \sum_{u^{\prime}=0}^{u-1} K_{u^{\prime}}}, \boldsymbol{\eta}_{u, L}\left(f_{u}^{w}, d_{u}\right), \mathbf{0}_{1 \times \sum_{u^{\prime}=u+1}^{U^{w}} K_{u^{\prime}}}\right]$, with $\boldsymbol{\eta}_{u, L}\left(f_{u}^{w}, d_{u}\right)=\left[\eta_{u, 1, L}\left(f_{u}^{w}, d_{u}\right), \ldots, \eta_{u, K_{q_{u}}, L}\left(f_{u}^{w}, d_{u}\right)\right] \in$ $\mathbb{C}^{1 \times K_{u}}$ denoting the complex gain of the LOS path between user $u$ and subarray $k \in \mathcal{K}_{q_{u}}$ of the AP in the subcarrier w. $K_{u}$ represents the number of antenna subarrays allocated 
to user $u$. Note that $\left|\eta_{u, k, L}\left(f_{u}^{w}, d_{u}\right)\right|, k=1, \ldots, K_{u}$ are the same and denoted as $\left|\eta_{u, L}\left(f_{u}^{w}, d_{u}\right)\right|$. Obviously, the equivalent channel $\tilde{\mathbf{h}}_{u}\left(f_{u}^{w}, d_{u}\right)$ approaches some determined vector as the number of subarray antennas goes to infinity.

With the above characterization, we first define $\boldsymbol{\Delta}$ and $\boldsymbol{\Xi}$ as

$$
\begin{gathered}
\boldsymbol{\Delta}=\operatorname{diag}\left(\left[\frac{1}{\left|\eta_{1,1, L}\right|}, \ldots, \frac{1}{\left|\eta_{1, K_{1}, L}\right|},\right.\right. \\
\left.\left.\ldots, \frac{1}{\left|\eta_{U^{w}, 1, L}\right|}, \ldots, \frac{1}{\left|\eta_{U^{w}, K_{U}, L}\right|}\right]\right), \\
\boldsymbol{\Xi}=\left[\begin{array}{llll}
\boldsymbol{\eta}_{1, L}^{H}\left(f_{1}^{w}, d_{1}\right) & \mathbf{0}_{K_{1} \times 1} & \cdots & \mathbf{0}_{K_{1} \times 1} \\
\mathbf{0}_{K_{2} \times 1} & \boldsymbol{\eta}_{2, L}^{H}\left(f_{2}^{w}, d_{2}\right) & \cdots & \mathbf{0}_{K_{2} \times 1} \\
\vdots & \vdots & \ddots & \vdots \\
\mathbf{0}_{K_{U} w 1} & \mathbf{0}_{K_{U} \times 1} & \cdots & \boldsymbol{\eta}_{U^{w}, L}^{H}\left(f_{U^{w}}^{w}, d_{U^{w}}\right)
\end{array}\right] .
\end{gathered}
$$

Then, we have the following result on the lower-bound of the achievable rate.

Theorem 1: When the number of subarray antennas approaches infinity, the lower-bound on the achievable rate of the indoor $\mathrm{THz}$ communication systems with finite-bit DACs is given by

$R^{o}=\sum_{u=1}^{U} \sum_{w \in \mathcal{W}_{u}} B \log _{2}\left(1+\frac{\frac{1}{\pi}\left|\mathbf{t}_{u}\left(f_{u}^{w}, d_{u}\right) \Delta \mathbf{G}_{L} \mathbf{t}_{u}^{H}\left(f_{u}^{w}, d_{u}\right)\right|^{2}}{\xi_{u}^{w}}\right)$,

where

$\mathbf{G}_{L}=\sum_{i=1}^{L_{D A C}-1} e^{-\frac{2}{K^{w} L_{D A C}^{2}}\left(i-\frac{L_{D A C}}{2}\right)^{2} \sum_{u \in \mathcal{U}}\left\|\boldsymbol{\eta}_{u, L}\right\|^{2} \operatorname{diag}\left(\boldsymbol{\Xi} \boldsymbol{\Xi}^{H}\right)^{-1}}$,

and

$$
\xi_{u}^{w}=\mathbf{t}_{u}\left(f_{u}^{w}, d_{u}\right) \mathbf{C}_{0} \mathbf{t}_{u}^{H}\left(f_{u}^{w}, d_{u}\right)
$$

with

$\mathbf{C}_{0}=2 \sum_{i=0}^{L_{D A C}-1}\left[\left(i-\frac{L_{D A C}}{2}+\frac{1}{2}\right)^{2}\left(\mathbf{\Phi}_{i+1}-\mathbf{\Phi}_{i}\right)\right]-\frac{1}{\pi} \mathbf{G}_{L} \mathbf{G}_{L}^{H}$,

where

$$
\boldsymbol{\Phi}_{i}=\Phi\left(\frac{2\left(i-\frac{L_{D A C}}{2}\right)}{L_{D A C}} \sqrt{\frac{\sum_{u \in \mathcal{U}^{w}}\left\|\boldsymbol{\eta}_{u, L}\right\|^{2}}{K^{w}}} \boldsymbol{\Delta}\right) .
$$

Proof: First, we can see from (9), (10), (11) and (30) that

$$
\mathbf{Q}_{M R T}=\mathbf{Q}_{Z F}=\sqrt{\frac{P^{w}}{\sum_{u \in \mathcal{U}^{w}}\left\|\boldsymbol{\eta}_{u, L}\right\|^{2}}} \mathbf{\Xi},
$$

where $\boldsymbol{\Xi}$ is defined in (32) when $M_{t}, N_{t}, M_{r}, N_{r} \rightarrow \infty$.

Substituting (38) into (14) gives us

$$
\dot{\mathbf{G}}=\sqrt{\frac{2}{\pi K^{w}\left(L_{D A C}-1\right)^{2}} \sum_{u \in \mathcal{U}^{w}}\left\|\boldsymbol{\eta}_{u, L}\right\|^{2}} \Delta \mathbf{G}_{L},
$$

where $\boldsymbol{\Delta}$ is defined in (31) and $\mathbf{G}_{L}$ is defined in (34).
Then, inserting (39) into (27) gives us

$\operatorname{diag}\left(\mathbf{C}_{x x}\right)$

$$
=2 \sum_{i=0}^{L_{D A C}-1} \frac{2 P^{w}}{K^{w}\left(L_{D A C}-1\right)^{2}}\left(i-\frac{L_{D A C}}{2}+\frac{1}{2}\right)^{2}\left(\boldsymbol{\Phi}_{i+1}-\boldsymbol{\Phi}_{i}\right),
$$

where $\boldsymbol{\Phi}_{i}$ in defined in (37).

Next, substituting (38)-(40) into (26), we can get

$$
\begin{aligned}
\mathbf{C}_{d d}^{d i a g} & =2 \sum_{i=0}^{L_{D A C}-1}\left[\frac{2 P^{w}}{K^{w}\left(L_{D A C}-1\right)^{2}}\left(i-\frac{L_{D A C}}{2}+\frac{1}{2}\right)^{2}\right. \\
& \left.\times\left(\boldsymbol{\Phi}_{i+1}-\boldsymbol{\Phi}_{i}\right)\right]-\frac{2 P^{w}}{\pi K^{w}\left(L_{D A C}-1\right)^{2}}\left(\mathbf{G}_{L} \mathbf{G}_{L}^{H}\right) .
\end{aligned}
$$

Finally, combining (38), (39) and (41) with (23), and defining $\xi_{u}^{w}$ in (35), we can arrive at (33) by noting that $\frac{N_{0}}{M_{t} N_{t} M_{r} N_{r}} \rightarrow 0$.

Corollary 1: For a special case of one-bit DACs, $R^{o}$ can be simplified as

$$
R_{b_{1}}^{o}=\sum_{u=1}^{U} \sum_{w \in \mathcal{W}_{u}} B \log _{2}\left(1+\frac{K_{u}^{2}\left|\eta_{u, L}\left(f_{u}^{w}, d_{u}\right)\right|^{2}}{\xi_{u, b_{1}}^{w}}\right),
$$

where $K_{u}$ represents the number of antenna subarrays allocated to user $u$ and $\xi_{u, b_{1}}^{w}$ can be expressed as

$$
\xi_{u, b_{1}}^{w}=\mathbf{t}_{u}\left(f_{u}^{w}, d_{u}\right) \mathbf{C}_{0}^{b_{1}} \mathbf{t}_{u}^{H}\left(f_{u}^{w}, d_{u}\right),
$$

with

$$
\begin{gathered}
\mathbf{C}_{0}^{b_{1}}=\arcsin \left(\operatorname{diag}\left(\boldsymbol{\Xi} \boldsymbol{\Xi}^{H}\right)^{-\frac{1}{2}} \Re\left\{\boldsymbol{\Xi} \boldsymbol{\Xi}^{H}\right\} \operatorname{diag}\left(\boldsymbol{\Xi} \boldsymbol{\Xi}^{H}\right)^{-\frac{1}{2}}\right) \\
+j \arcsin \left(\operatorname{diag}\left(\boldsymbol{\Xi} \boldsymbol{\Xi}^{H}\right)^{-\frac{1}{2}} \Im\left\{\boldsymbol{\Xi} \boldsymbol{\Xi}^{H}\right\} \operatorname{diag}\left(\boldsymbol{\Xi} \boldsymbol{\Xi}^{H}\right)^{-\frac{1}{2}}\right) \\
-\boldsymbol{\Delta} \boldsymbol{\Xi} \boldsymbol{\Xi}^{H} \boldsymbol{\Delta}^{H} .
\end{gathered}
$$

Remark 2: As indicated in the proof, the inter-user interference can be eliminated by the analog transmit beamforming, where different sets of subarrays are allocated to different groups of users in the large subarray antenna regime and the data stream for each user can be forwarded solely to the allocated subarrays. More specifically, we will have singleuser transmission with the allocated subarrays of antennas in the allocated transmission window for each user.

Remark 3: Note also that (33) is a constant and is independent of the transmit power $P$. This tells us that the $\mathrm{THz}$ communication systems with finite-bit DACs should work in the low power regime to be power efficient when the number of antennas in each subarray goes to infinity or is very large since increasing transmit power doesnot improve the performance. Moreover, the major error source is due to the DACs rather than the AWGN at the receiver.

\section{B. Finite-bit ADCs Only}

Consider the indoor $\mathrm{THz}$ communication system only with finite-bit ADCs. According to (18) and let $\mathbf{q}_{u}$ be the $u$-th column of the precoding matrix $\mathbf{Q}$, the quantized received signal $\ddot{y}_{u}$ after receiver analog beamforming at user $u$ in group 
$q$ in subcarrier $w$ can be described as

$$
\begin{aligned}
\ddot{y}_{u} & =\left(1-e_{q}\right) \tilde{\mathbf{h}}_{u}\left(f_{u}^{w}, d_{u}\right) \mathbf{q}_{u} s_{u} \\
& +\left(1-e_{q}\right) \sum_{u^{\prime} \in \mathcal{U}^{w}, u^{\prime} \neq u} \tilde{\mathbf{h}}_{u}\left(f_{u}^{w}, d_{u}\right) \mathbf{q}_{u^{\prime}} s_{u^{\prime}} \\
& +\left(1-e_{q}\right) n_{u}+\varpi_{u},
\end{aligned}
$$

where $\mathbf{q}_{u} \in \mathbb{C}^{K^{w} \times 1}$ denotes the digital precoding vector corresponding to user $u$, and $n_{u}$ is the AWGN with power $N_{0}$ for user $u$. $\varpi_{u}$ is the $u$ th entry of the distortion error $\varpi$.

Then, we know that the achievable rate of user $u$ is lowerbounded by [33]

$\ddot{R}_{u} \geq \mathbb{E}\left[\sum_{w \in \mathcal{W}_{u}} B \log _{2}(1+\right.$

$\left.\left.\frac{\left(1-e_{q}\right)^{2}\left|\tilde{\mathbf{h}}_{u}\left(f_{u}^{w}, d_{u}\right) \mathbf{q}_{u}\right|^{2}}{\sum_{u \neq u^{\prime}}\left(1-e_{q}\right)^{2}\left|\tilde{\mathbf{h}}_{u}\left(f_{u}^{w}, d_{u}\right) \mathbf{q}_{u^{\prime}}\right|^{2}+\mathbb{E}\left\{\left|\varpi_{u}\right|^{2}\right\}+\left(1-e_{q}\right)^{2} N_{0}}\right)\right]$

where

$$
\begin{aligned}
& \mathbb{E}\left\{\left|\varpi_{u}\right|^{2}\right\}=e_{q}\left(1-e_{q}\right) \mathbb{E}\left\{\operatorname{diag}\left(\dddot{\mathbf{r i}}^{H}\right)_{u, u}\right\} \\
& =e_{q}\left(1-e_{q}\right) \mathbb{E}\left\{\operatorname{diag}\left((\tilde{\mathbf{H} Q \mathbf{Q}}+\mathbf{n})(\tilde{\mathbf{H}} \mathbf{Q} \mathbf{s}+\mathbf{n})^{H}\right)_{u, u}\right\} \\
& =e_{q}\left(1-e_{q}\right) \mathbb{E}\left\{\tilde{\mathbf{h}}_{u}\left(f_{u}^{w}, d_{u}\right)\left(\mathbf{Q s s} \mathbf{Q}^{H} \mathbf{Q}^{H}\right) \tilde{\mathbf{h}}_{u}^{H}\left(f_{u}^{w}, d_{u}\right)+\left|n_{u}\right|^{2}\right\} \\
& =e_{q}\left(1-e_{q}\right)\left[\sum_{u^{\prime}}\left|\tilde{\mathbf{h}}_{u}\left(f_{u}^{w}, d_{u}\right) \mathbf{q}_{u^{\prime}}\right|^{2}+N_{0}\right]
\end{aligned}
$$

where (47) is derived with $\mathbb{E}\left\{\mathbf{s s}^{H}\right\}=\mathbf{I}_{U^{w}}$.

Finally, we can write the sum rate of the indoor $\mathrm{THz}$ communication systems only with finite-bit ADCs as

$$
\ddot{R}=\sum_{u=1}^{U} \ddot{R}_{u}
$$

Considering the indoor $\mathrm{THz}$ communication systems with infinite-bit DACs and finite-bit ADCs at the receiver. From proposition 1, the analog beamforming angles of each user group are mainly decided by the AoD/AoA of the LOS path in large subarray antenna regime. So that we have the following result on the lower-bound of the achievable rates.

Theorem 2: When the number of subarray antennas approaches infinity, the closed-form lower-bound of the achievable rate of the indoor $\mathrm{THz}$ communication system with finitebit ADCs is given by

$$
R^{o}=\sum_{u=1}^{U} W_{u} B \log _{2}\left(1+\frac{1-e_{q}}{e_{q}}\right)
$$

where $e_{q} \approx \frac{\pi \sqrt{3}}{2} 2^{-2 b}$ and $W_{u}$ is the total number of subcarriers for user $u$.

Proof: First, we can see from (9), (10), (11) and (30) that

$$
\mathbf{Q}_{M R T}=\mathbf{Q}_{Z F}=\frac{\sqrt{P^{w}}}{\sqrt{\sum_{u \in \mathcal{U}^{w}}\left\|\boldsymbol{\eta}_{u, L}\right\|^{2}}} \boldsymbol{\Xi},
$$

where $\Xi$ is defined in (32). Then, (48) can be rewritten as

$$
E\left\{\left|\varpi_{u}\right|^{2}\right\}=e_{q}\left(1-e_{q}\right)\left[M_{t} N_{t} M_{r} N_{r} \Omega_{t}^{2} \Omega_{r}^{2} K_{u}^{2}\left|\eta_{u, L}\left(f_{u}^{w}, d_{u}\right)\right|^{2}+N_{0}\right] .
$$

Incorporating (46), (51)-(52) and after simple computations, we derive (50) by noting that $\frac{N_{0}}{M_{t} N_{t} M_{r} N_{r}} \rightarrow 0$.

Remark 4: It is interesting that the transmission rate with finite-bit ADCs at user sides is robust to the transmit power and mainly depends on the distortion parameters. Obviously, when $b$ increases, i.e., higher-resolution ADCs, $e_{q}$ decreases and hence the achievable rate $R^{o}$ increases.

Remark 5: When the number of subarray antennas goes to infinity, the Gaussian noise can be ignored and the interference source is mainly the quantized distortion. Besides, the interference among user groups is eliminated due to the strategy of allocating different number of subarrays to different user groups and the strong directionality of the large antenna subarray.

\section{The System With Both Finite-bit DACs and Finite-bit ADCs}

Considering the case that the indoor $\mathrm{THz}$ system with finitebit DACs and finite-bit ADCs at the receiver. Incorporating (13) and (18), the output data vector can be decomposed into two uncorrelated components [24] and given by

$$
\mathbf{y}_{q}=\ddot{\mathbf{G}}(\tilde{\mathbf{H}} \mathbf{x}+\mathbf{n})+\mathbf{n}_{A D},
$$

where $\ddot{\mathbf{G}}$ stands for the ADC operation and defined in (19). $\mathbf{n} \in \mathbb{C}^{U^{w} \times 1}$ is the AWGN vector. And the distortion caused by the quantized $\mathrm{ADC}$ is given by

$$
\mathbb{E}\left\{\mathbf{n}_{A D} \mathbf{n}_{A D}^{H}\right\}=e_{q}\left(1-e_{q}\right) \mathbb{E}\left\{\operatorname{diag}\left((\tilde{\mathbf{H}} \mathbf{x}+\mathbf{n})(\tilde{\mathbf{H}} \mathbf{x}+\mathbf{n})^{H}\right)\right\},
$$

where $e_{q} \approx \frac{\pi \sqrt{3}}{2} 2^{-2 b}$ is the distortion factor of quantized ADCs.

Then, the received signal for user $u$ can be obtained by

$$
\begin{aligned}
y_{q u} & =\left(1-e_{q}\right) \tilde{\mathbf{h}}_{u}\left(f_{u}^{w}, d_{u}\right) \dot{\mathbf{G}} \mathbf{q}_{u} s_{u} \\
& +\left(1-e_{q}\right) \sum_{u^{\prime} \in \mathcal{U}^{w}, u^{\prime} \neq u} \tilde{\mathbf{h}}_{u}\left(f_{u}^{w}, d_{u}\right) \dot{\mathbf{G}} \mathbf{q}_{u^{\prime}} s_{u^{\prime}} \\
& +\left(1-e_{q}\right) \tilde{\mathbf{h}}_{u}\left(f_{u}^{w}, d_{u}\right) \mathbf{d}+\left(1-e_{q}\right) n_{u}+n_{A D, u},
\end{aligned}
$$

and then the achievable rate lowerbound of user $u$ with finitebit DACs and quantized ADCs can be written as (56) on top of next page, where

$$
\begin{aligned}
\mathbb{E}\left\{\left|n_{A D, u}\right|^{2}\right\} & =e_{q}\left(1-e_{q}\right)\left(\sum_{i}\left|\tilde{\mathbf{h}}_{u}\left(f_{u}^{w}, d_{u}\right) \dot{\mathbf{G}} \mathbf{q}_{i}\right|^{2}\right. \\
& \left.+\tilde{\mathbf{h}}_{u}\left(f_{u}^{w}, d_{u}\right) \mathbf{C}_{d d} \tilde{\mathbf{h}}_{u}^{H}\left(f_{u}^{w}, d_{u}\right)+N_{0}\right) .
\end{aligned}
$$

Thus, when the indoor $\mathrm{THz}$ communication systems with both finite-bit precoding and finite-bit ADCs at the user sides, the sum rate is

$$
R=\sum_{u=1}^{U} R_{q u}
$$

Theorem 3: When the number of subarray antennas approaches infinity, the closed-form lower-bound of the achievable rate of the indoor $\mathrm{THz}$ communication system with finitebit DACs and finite-bit ADCs is given by

$R^{o}=\sum_{u=1}^{U} \sum_{w \in \mathcal{W}_{u}} B \log _{2}\left(1+\frac{\left(1-e_{q}\right) \frac{1}{\pi}\left|\mathbf{t}_{u}\left(f_{u}^{w}, d_{u}\right) \Delta \mathbf{G}_{L} \mathbf{t}_{u}^{H}\left(f_{u}^{w}, d_{u}\right)\right|^{2}}{e_{q} \frac{1}{\pi}\left|\mathbf{t}_{u}\left(f_{u}^{w}, d_{u}\right) \mathbf{\Delta} \mathbf{G}_{L} \mathbf{t}_{u}^{H}\left(f_{u}^{w}, d_{u}\right)\right|^{2}+\xi_{u}^{w}}\right)$, 
$R_{q u} \geq \mathbb{E}\left[\sum_{w \in \mathcal{W}_{u}} B \log _{2}\left(1+\frac{\left(1-e_{q}\right)^{2}\left|\tilde{\mathbf{h}}_{u}\left(f_{u}^{w}, d_{u}\right) \dot{\mathbf{G}} \mathbf{q}_{u}\right|^{2}}{\left(1-e_{q}\right)^{2} \sum_{u \neq i}\left|\tilde{\mathbf{h}}_{u}\left(f_{u}^{w}, d_{u}\right) \dot{\mathbf{G}} \mathbf{q}_{i}\right|^{2}+\left(1-e_{q}\right)^{2} \tilde{\mathbf{h}}_{u}\left(f_{u}^{w}, d_{u}\right) \mathbf{C}_{d d} \tilde{\mathbf{h}}_{u}^{H}\left(f_{u}^{w}, d_{u}\right)+\left(1-e_{q}\right)^{2} N_{0}+\mathbb{E}\left\{\left|n_{A D, u}\right|^{2}\right\}}\right)\right]$

where $\xi_{u}^{w}$ is described in (35) and $e_{q}$ is defined in (21).

Proof: The idea is similar to the proof of theorem 1 and theorem 2 and is omitted here for brevity.

Corollary 2: For the case of one-bit DACs and finite-bit ADCs,

$$
R_{b_{1}}^{o}=\sum_{u=1}^{U} \sum_{w \in \mathcal{W}_{u}} B \log _{2}\left(1+\frac{\left(1-e_{q}\right) K_{u}^{2}\left|\eta_{u, L}\left(f_{u}^{w}, d_{u}\right)\right|^{2}}{e_{q} K_{u}^{2}\left|\eta_{u, L}\left(f_{u}^{w}, d_{u}\right)\right|^{2}+\xi_{u, b_{1}}^{w}}\right)
$$

where $\xi_{u, b_{1}}^{w}$ is described in (43) and $e_{q}$ is defined in (21).

Remark 6: Undoubtedly, the transmission rate is independent of transmit power $P$ and related to the distortion factor $e_{q}$ and $\xi_{u}^{w}$. It reveals that the performance of indoor $\mathrm{THz}$ system with finite-bit precoding and low-resolution ADCs at the receiver will be stable when the number of subarray antennas is large enough. Besides, the main interference source is mainly the quantized distortion caused by low-resolution DACs and ADCs.

\section{Single-User Case}

As indicated in Remark 2 with the adopted transmission scheme, we can treat the data transmission to each user as a single-user transmission with the allocated subarrays of antennas. In the following, we consider the single-user case.

Suppose that $P^{w}=\frac{P}{W}$ with $W$ representing the total number of subcarriers for user $u$. Note that the lower-bound on the achievable rate can be expressed similar to (50). Here, we consider a simple case and can derive the following result regarding the single-user case.

Corollary 3: In the single-user indoor $\mathrm{THz}$ communication systems with finite-bit precoding at the AP, the lower-bound on the achievable rate for the user with $\psi_{L}^{r}=\phi_{L}^{r}=\psi_{L}^{t}=\phi_{L}^{t}=0$ in the large subarray antenna regime is given by

$$
R_{u=1}^{o}=B W \log _{2}\left(1+\frac{\alpha}{\pi \beta}\right)
$$

where

$$
\begin{gathered}
\alpha=\sum_{i=1}^{L_{D A C}-1} e^{-\frac{2\left(i-\frac{L_{D A C}}{2}\right)^{2}}{L_{D A C}^{2}}} \\
\beta=2 \sum_{i=0}^{L_{D A C}-1}\left\{( i - \frac { L _ { D A C } } { 2 } + \frac { 1 } { 2 } ) ^ { 2 } \left[\Phi\left(\frac{2\left(i+1-\frac{L_{D A C}}{2}\right)}{L_{D A C}}\right)\right.\right. \\
\left.\left.-\Phi\left(\frac{2\left(i-\frac{L_{D A C}}{2}\right)}{L_{D A C}}\right)\right]\right\}-\frac{\alpha^{2}}{\pi} .
\end{gathered}
$$

For the case of one-bit DACs, $R_{u=1, b=1}^{o}$ can be expressed as

$$
R_{u=1, b=1}^{o}=B W \log _{2}\left(1+\frac{1}{\frac{\pi}{2}-1}\right)
$$

Proof: Note that with the assumption on the AoD/AoA, we know that $\eta_{u, k, L}\left(f_{u}^{w}, d_{u}\right)=\eta_{u, L}\left(f_{u}^{w}, d_{u}\right), \forall k$, i.e., $\boldsymbol{\eta}_{u, L}\left(f_{u}^{w}, d_{u}\right)=\eta_{u, L}\left(f_{u}^{w}, d_{u}\right) \mathbf{1}_{1 \times K}$. Now, $\mathbf{G}_{L}$ in (34) for one user with the updated $\boldsymbol{\eta}_{u, L}\left(f_{u}^{w}, d_{u}\right)$ can be rewritten as

$$
\mathbf{G}_{L}^{u=1}=\alpha \mathbf{1}_{K \times K}
$$

Then, from (36), we can obtain

$$
\mathbf{C}_{0}^{u=1}=\beta \mathbf{1}_{K \times K} .
$$

Moreover, $\mathbf{C}_{0}^{u=1, b=1}=\left(1-\frac{2}{\pi}\right) \mathbf{1}_{K \times K}$ is for the case of $b_{D A C}=1$. Therefore, we have

$$
\begin{aligned}
\xi_{u=1}^{w} & =\boldsymbol{\eta}_{u, L}\left(f_{u}^{w}, d_{u}\right) \mathbf{C}_{0}^{u=1} \boldsymbol{\eta}_{u, L}^{H}\left(f_{u}^{w}, d_{u}\right), \\
& =\beta\left|\eta_{u, L}\left(f_{u}^{w}, d_{u}\right)\right|^{2} K^{2} .
\end{aligned}
$$

Considering the rate expression in (33), (65) and (67), we have the result in (61).

Remark 7: This tells us that the achievable rate for the user with properly placed antennas, i.e.zero AoD/AoA angles, is insensitive to the distance between the AP and the number of subarrays in the large subarray antenna regime. That is, with the one-bit DACs, increasing the number of subarrays may not improve the spectral efficiency for certain user since the distortion introduced by the finite-bit DACs increases with the number of subarrays as well.

Corollary 4: For the single-user case when the system with finite-bit precoding and finite-bit ADCs at the receiver. Similarly, we can obtain the lower-bound on the achievable rate as

$$
R^{o}=B W \log _{2}\left(1+\frac{\frac{\left(1-e_{q}\right) \alpha}{\pi}}{\frac{e_{q} \alpha}{\pi}+\beta}\right) .
$$

Remark 8: Apparently, the achievable rate is a constant when the number of subarray antenna approaches to infinity, which also reveals that increasing the transmission power or the number of antenna subarrays does not introduce much benefit when the number of subarray antennas is large enough. This is due to the large distortion caused by the low-resolution DACs and ADCs.

In addition, there may be phase uncertainties for the $\mathrm{THz}$ band, which will degrade the achievable rate [13]. Denote $\sigma_{m_{t}, n_{t}}(f)$ and $\sigma_{m_{r}, n_{r}}(f)$ as the random phase errors at the transmitter and receiver, respectively. Assume that $\sigma_{m_{t}, n_{t}}(f)$ and $\sigma_{m_{r}, n_{r}}(f)$ follow uniform distribution in $\left[-\varepsilon_{t}(f), \varepsilon_{t}(f)\right]$ and $\left[-\varepsilon_{r}(f), \varepsilon_{r}(f)\right]$, respectively. Then, we have the following proposition.

Proposition 2: When the number of antennas in each subarray goes to infinity, the rate loss due to the phase uncertainties with uniform distributions can be completely ignored in the indoor $\mathrm{THz}$ communications with finite-bit DACs and/or finitebit ADCs.

Proof: See Appendix B for details. 


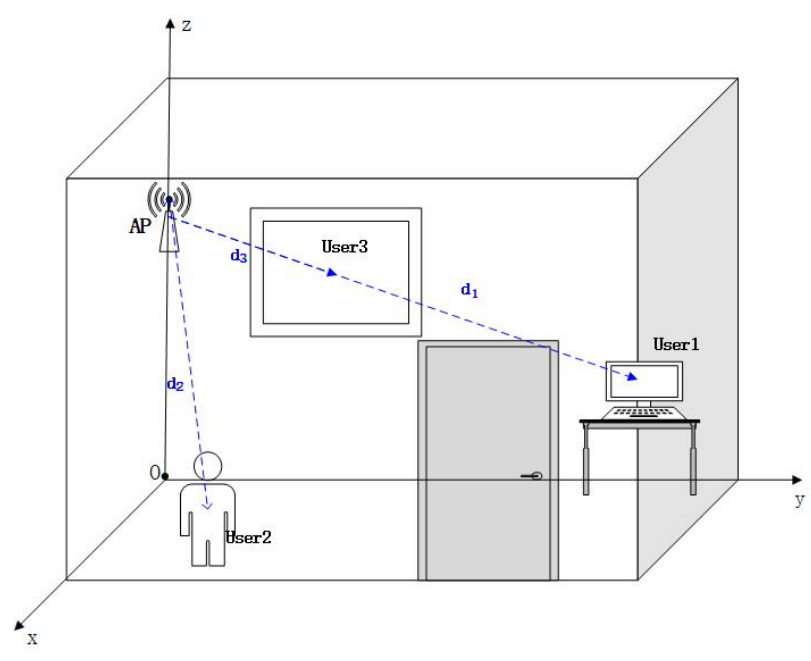

Fig. 2. Simulation scenario for indoor $\mathrm{THz}$ communication.

Remark 9: This is generally different from the previous study that rate loss will increase as the number of antennas increases. The main reason is that the distortion error caused by coarse quantization dominates the system performance.

\section{Numerical Results}

In this section, we numerically evaluate the performance of the indoor $\mathrm{THz}$ communication systems with finite-bit DACs at the transmitter or finite-bit ADCs at the receiver. The simulation parameters are given in Tables I and II. The relative locations of the users and AP can be seen in Fig. 2 for example. Specifically, the transmission windows for user 1 and user 3 are not overlapping with each other, and hence grouping them together will not introduce any interference since orthogonal transmissions for the users can be expected. As shown in [11], the coherence bandwidth may take values from $1 \mathrm{GHz}$ to 5 GHz. In the following numerical evaluations, the subband bandwidth is set to be $B=5 \mathrm{GHz}$, and hence the transmission window for each user is composed of $W=50$ subbands. In addition, we assume the same size for each subarray, i.e., $M_{t}=N_{t}=M_{r}=N_{r}$. In order to enable us to use the diagonal approximation in multi-bit DACs, the clipping level $A_{c}$ can be set as $A_{c}=\sqrt{\frac{P}{2 K}}\left(1-\Phi^{-1}\left(P_{c} / 2\right)\right)$, where $P_{c}$ is the probability of clipping the signal in the DACs. Here, we set $P_{c}=0.1 \%$ as [22]. Without loss of generality, we assume $K_{1}=5$ and $K_{2}=3$, i.e., 5 subarrays are allocated to group 1 while 3 subarrays are allocated to group 2 . We would like to note that the allocation of the subarrays will affect the achievable rate but not the trend of the curves, and the choice of $K_{1}=5$ and $K_{2}=3$ can achieve the largest rate. On the other hand, in case of single-user case, we take user 1 as an example and $K_{1}=8$.

\section{A. Finite-bit DACs Only}

Fig. 3 considers the indoor $\mathrm{THz}$ communication system with one-bit DACs at the transmitter and infinite-bit ADCs at the receiver, which shows the achievable rate with different analog beamforming method as the transmit power increases
TABLE I

TRANSMISSION WINDOWS

\begin{tabular}{|c|c|c|c|}
\hline & Distance & $\begin{array}{c}\text { Transmission } \\
\text { Windows (THz) }\end{array}$ & User Group \\
\hline User1 & $10 \mathrm{~m}$ & $\begin{array}{c}0.6-0.7, \\
0.8-0.95\end{array}$ & group1 \\
\hline User2 & $5 \mathrm{~m}$ & $\begin{array}{c}0.6-0.725, \\
0.8-0.925\end{array}$ & group2 \\
\hline User3 & $1 \mathrm{~m}$ & $\begin{array}{c}0.5-0.6, \\
0.7-0.8,0.95-1\end{array}$ & group1 \\
\hline
\end{tabular}

TABLE II

SYSTEM PARAMETERS

\begin{tabular}{|c|c|c|}
\hline Definitions & Parameters & Values \\
\hline Antenna gain & $\Omega_{t}, \Omega_{r}$ & $20 \mathrm{dBi}, 20 \mathrm{dBi}$ \\
\hline Subcarrier bandwidth & $\mathrm{B}$ & $5 \mathrm{GHz}$ \\
\hline Total number of subarrays & $\mathrm{K}$ & 8 \\
\hline Noise power & $N_{0}$ & $-75 \mathrm{dBm}$ \\
\hline Maximum phase uncertainties & $\varepsilon_{t}(f), \varepsilon_{r}(f)$ & $\frac{\pi}{18}, \frac{\pi}{18}$ \\
\hline
\end{tabular}

for different subarray antenna size in the single-user case. We take user 1 as an example. We choose the analog beamforming angles either through codebook, i.e., (5), or the direction of the LOS path, i.e., (29). From the figure, as the transmit power increases, the curves with finite subarray size approach the constant value achieved with $M_{t}, N_{t}, M_{r}, N_{r} \rightarrow \infty$, which is independent of transmission power. Also, we can see that as the number of antennas in each subarray increases, the gap between the achievable rate with the analog beamforming specified by the LOS path and the one achieved by the codebook diminishes. More specifically, we can see from the figure that while the achievable rate with $16 \times 16$ antenna subarrays can get close to the infinite case at around $P=-20$ $\mathrm{dBm}$, the $8 \times 8$ and $4 \times 4$ antenna subarrays can only get close to the infinite case at around $P=-10 \mathrm{dBm}$ and $P=1 \mathrm{dBm}$, respectively. Hence, the achievable rate with $16 \times 16$ antenna subarrays can approach the limitation in a lower transmission power regime. In the following, we assume that the analog beamforming angles are specified by the AoD/AoA of the LOS path.

Fig. 4 shows the achievable rate of the system, i.e., the sum rate (33) for the considered 3 users case, with different resolutions of DACs when the system is equipped with finitebit DACs at the transmitter and infinite-bit ADCs at the receiver. We assume $16 \times 16$ subarray size. From the figure, we can see that when the DAC resolution increases, i.e., the quantization bits $b$ increases, the transmission rate increases as well. Nearly the same performance can be achieved as the unquantized transmission rate at moderate DAC resolution, i.e., $b=5,8$, in the low-power regime. This tells us that the system with finite-bit DACs can achieve performance close to the infinite-resolution one so that the hardware complexity and cost can be excessively reduced. 


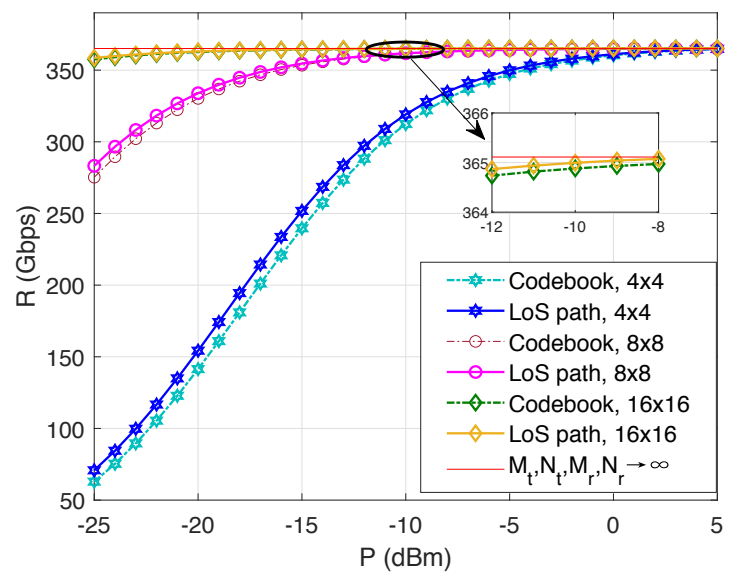

Fig. 3. Achievable rate of user 1 with different analog beamforming methods and antenna array sizes in the 3 users case as $P$ varies. One-bit DACs only.

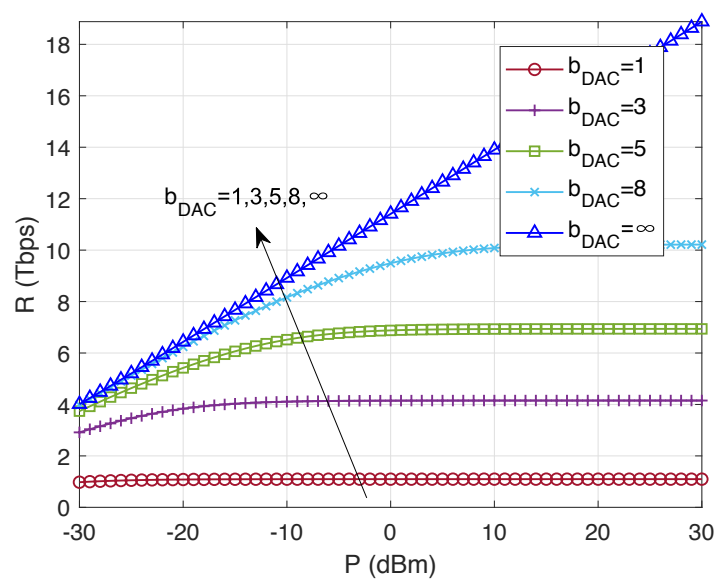

Fig. 4. Achievable rate with different resolutions of DACs in the 3 users case as $P$ varies. Finite-bit DACs only.

\section{B. Finite-bit ADCs Only}

Fig. 5 plots the achievable rate of the system, i.e., the sum rate (50) for the considered 3 users case, with different resolutions of ADCs when the system is only equipped with finite-bit $\mathrm{ADCs}$ at the receiver. We assume $16 \times 16$ subarray size. From the figure, we can see that the quantized transmission rate increases as $\mathrm{ADC}$ resolution increases, i.e., $b$ increases, and can achieve almost the same performance as the unquantized transmission rate in the low-power regime when $b=5,8$, which again tells us that moderate resolution ADCs can be applied to the indoor $\mathrm{THz}$ communications in the low-power regime.

Fig. 6 considers the indoor $\mathrm{THz}$ communication system for single user only with one-bit DACs or one-bit ADCs, respectively. We take user 1 as an example. It is shown that the rate loss caused by the low-resolution ADCs is larger than DACs with the same resolution. Moreover, increasing the number of subarray antennas might not compensate for the large loss for the low-resolution ADCs/DACs.

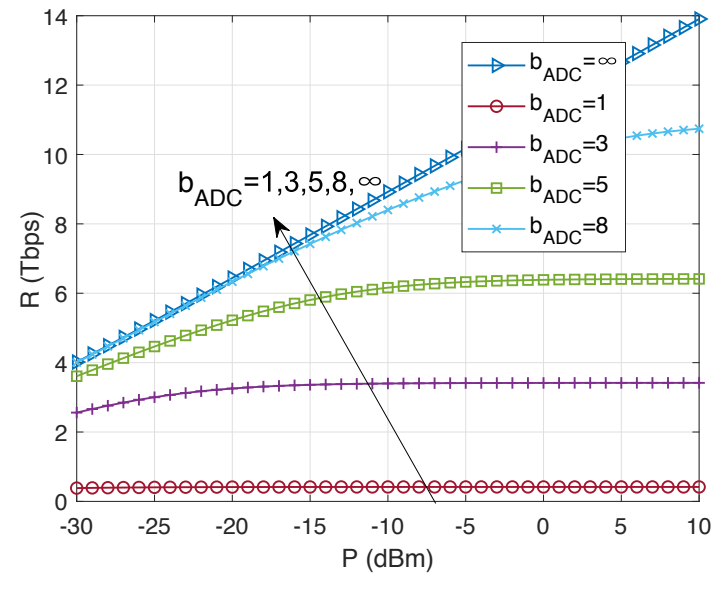

Fig. 5. Achievable rate with different resolutions of ADCs in the 3 users case as $P$ varies. Finite-bit ADCs only.

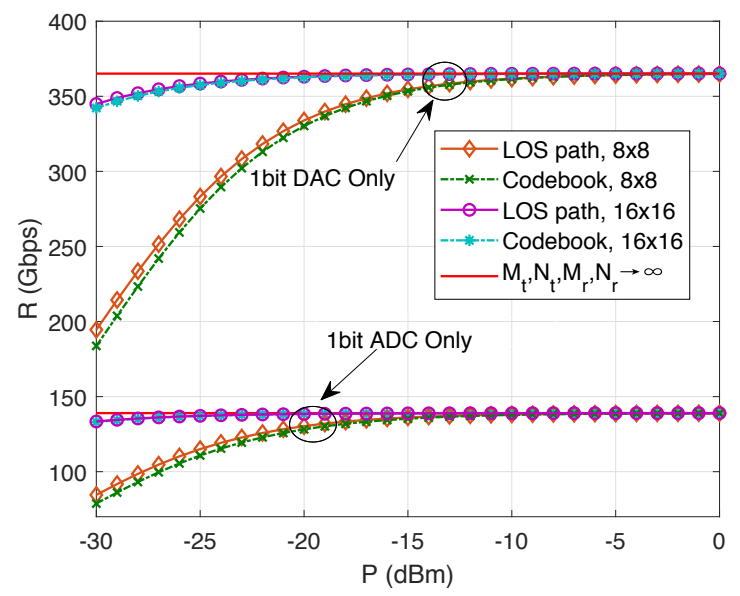

Fig. 6. Achievable rate of user 1 in the 3 users case as $P$ varies. One-bit DACs only or one-bit ADCs only.

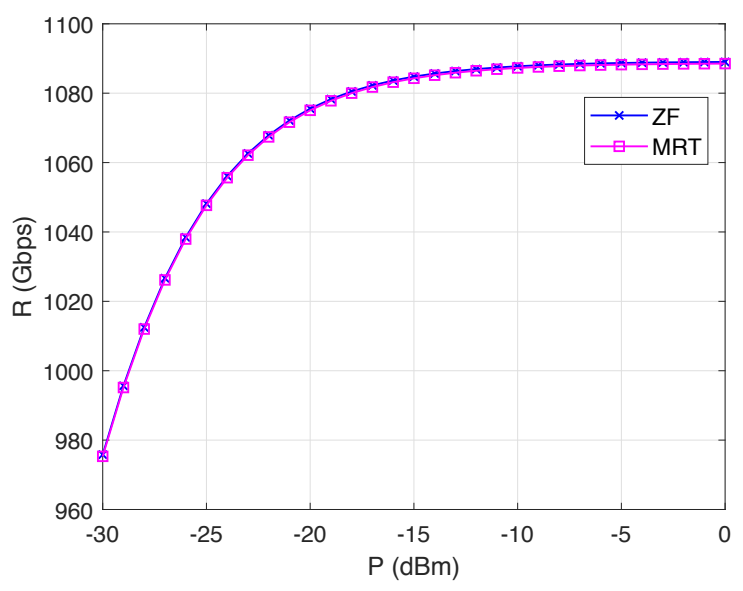

Fig. 7. Achievable rate of the MRT and ZF schemes in the 3 users case as $P$ varies. Both one-bit DACs and 5-bit ADCs. 


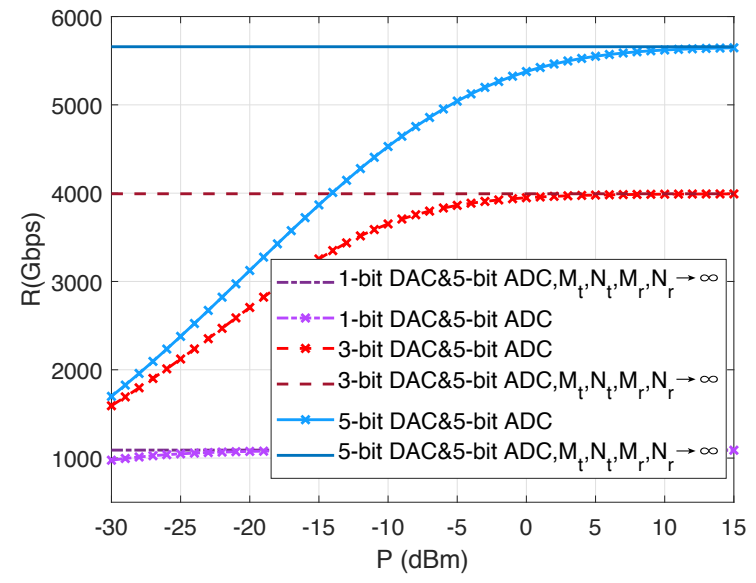

(a) Achievable rate with finite-bit DACs and 5-bit ADCs.

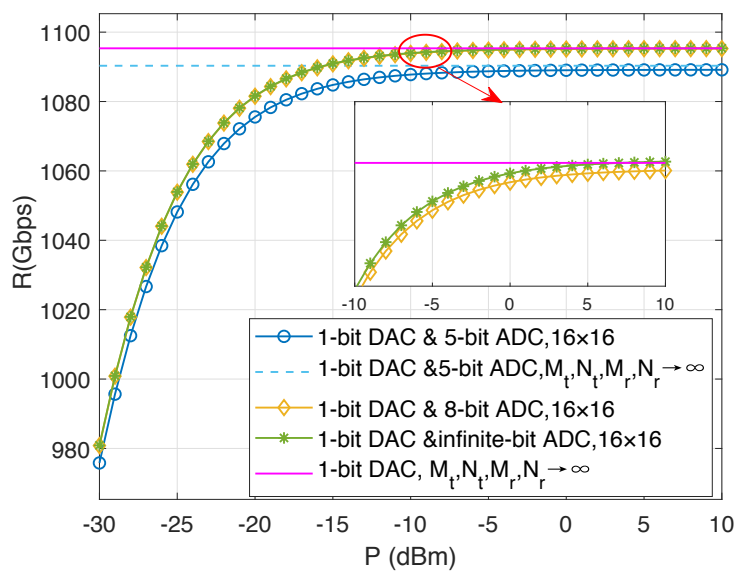

(b) Achievable rate with one-bit DACs and finite-bit ADCs.

Fig. 8. Achievable rate with different resolutions of DACs and ADCs in the 3 users case as $P$ varies. Both finite-bit DACs and finite-bit ADCs.

\section{Both Finite-bit DACs and Finite-bit ADCs}

In Fig. 7, we plot the achievable rate of the system, i.e., the sum rate (58) for the considered 3 users case, when the system is equipped with one-bit DACs and 5-bit ADCs. We assume $16 \times 16$ subarray size. From the figure, we can find that the performance gap between $\mathrm{ZF}$ and MRT vanishes, which aligns with Theorem 3.

In Figs. 8(a) and 8(b), we plot the achievable rate with $M_{t}=N_{t}=M_{r}=N_{r}=16$ for the multi-user case when the system is equipped with finite-bit DACs at the transmitter and 5-bit ADCs at the receiver, and one-bit DACs at the transmitter and finite-bit ADCs at the receiver, respectivley. From the figures, we can see that the achievable rate of different resolution DACs and ADCs reaches a constant independent of the transmit power with $M_{t}, N_{t}, M_{r}, N_{r} \rightarrow \infty$, verifying the analysis. Again, we can find that finite-resolution ADCs introduces more rate loss and DACs.

Fig. 9 considers the system with finite-bit DACs at the transmitter and 5-bit ADCs at the receiver, we plot the achievable rate with different resolution of DACs for the single-user with $\psi_{L}^{r}=\phi_{L}^{r}=\psi_{L}^{t}=\phi_{L}^{t}=0$ and $K=8$. Interestingly, at moderate resolution DACs and ADCs, e.g., 5-bit DACs

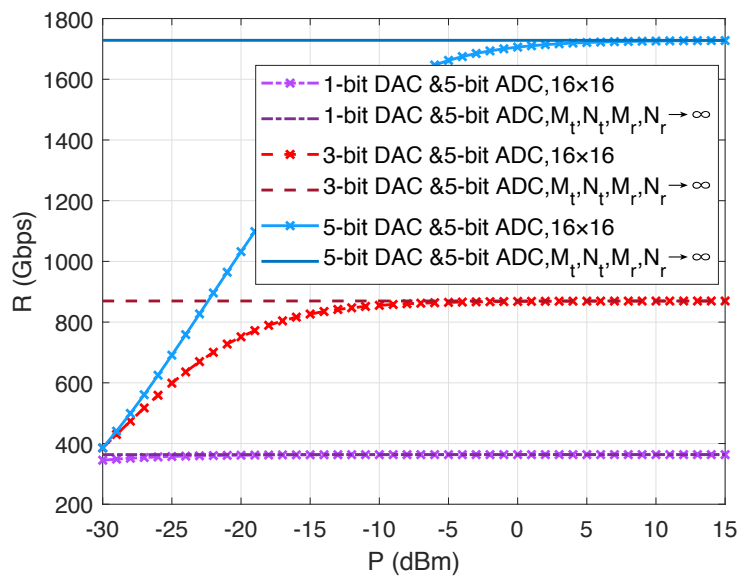

Fig. 9. Achievable rate with $\psi_{L}^{r}=\phi_{L}^{r}=\psi_{L}^{t}=\phi_{L}^{t}=0$ in the single-user case as $P$ varies. Both finite-bit DACs and 5-bit ADCs.

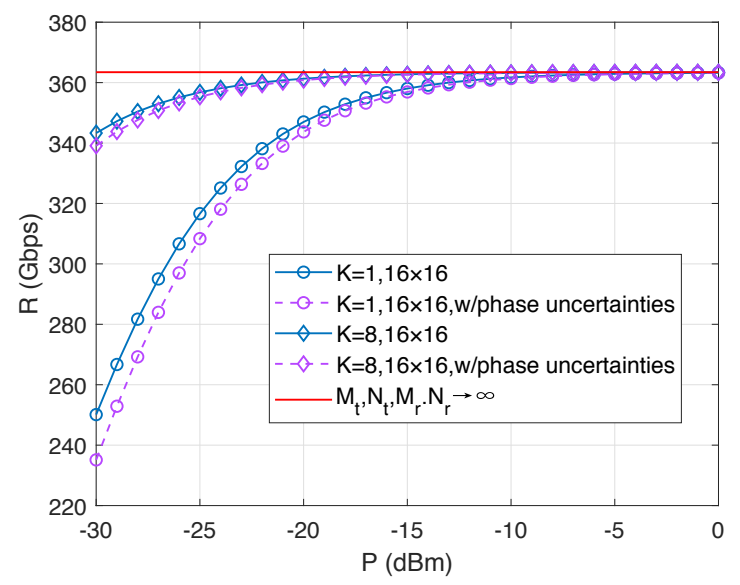

Fig. 10. Impact of uniform phase error uncertainties on the achievable rate with $\psi_{L}^{r}=\phi_{L}^{r}=\psi_{L}^{t}=\phi_{L}^{t}=0$ in the single-user case as $P$ varies. Both one-bit DACs and 5-bit ADCs.

and 5-bit ADCs, we can achieve Tbps for the indoor $\mathrm{THz}$ communication systems.

Fig. 10 considers the system with one-bit DACs at the transmitter and 5-bit ADCs at the receiver, we plot the achievable rate with different numbers of antenna subarrays in the presence of phase uncertainties for the single-user with $\psi_{L}^{r}=\phi_{L}^{r}=\psi_{L}^{t}=\phi_{L}^{t}=0$. It can be seen from the figure that the different curves approach the same value achieved with $M_{t}, N_{t}, M_{r}, N_{r} \rightarrow \infty$ regardless of the phase uncertainties, i.e., the impact of phase uncertainties on the achievable rate can be negligible in the large subarray antenna regime. Then, in case of phase errors with uniform distributions, increasing the number of subarrays may not be helpful when the number of the antennas in each subarray is large enough.

Fig. 11 considers the system with 1-bit DACs at the transmitter and 5-bit ADCs at the receiver with the antenna number $M_{t}=N_{t}=M_{r}=N_{r}$. We plot the achievable rate with different number of antennas for multi-users with the transmit power $P=0 \mathrm{dBm}$ and $P=-10 \mathrm{dBm}$. It can be seen that with the increasing of antenna numbers, the achievable rate tends to be stable. Moreover, increasing the number of antennas might 


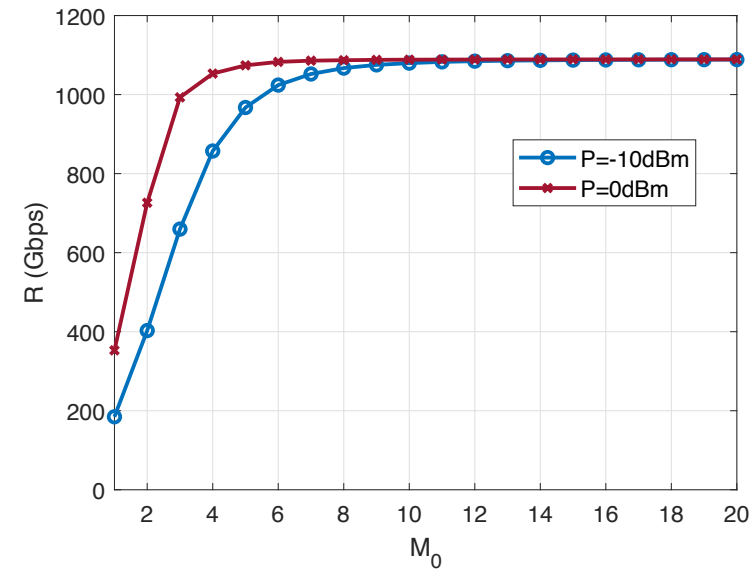

Fig. 11. Achievable rate with different number of antennas with $P=0$ $\mathrm{dBm}$ and $P=-10 \mathrm{dBm}$ in the 3 users case. Both one-bit DACs and 5-bit ADCs.

not reach higher achievable rate, which again aligns with the findings in Theorem 3, where the achievable rate saturates and is unrelated to the transmit power as the number of subarray antennas approaches infinity due to the quantization distortions cause by low-resolution DACs and ADCs.

\section{CONCLUSIONS}

In this paper, we have considered the downlink indoor $\mathrm{THz}$ communication system with low-resolution DACs or ADCs. We have assumed that distance-aware multi-carrier strategy and antenna-of-subarrays architecture with hybrid precoding scheme are employed. We have proved that the analog beamforming angles for each group of users are decided by the LOS path in the large subarray antenna regime. We have derived the closed-form expression for the lower bound on the achievable rate, which does not change with the transmit power when the number of the subarray antennas goes to infinity. Through numerical results, we have shown that the transmission rate with moderate resolution DACs and ADCs can achieve almost the same performance as the infinite resolution one. We have found that low-resolution ADCs cause more rate loss than DACs in the $\mathrm{THz}$ communication system. Interestingly, we have demonstrated that the indoor $\mathrm{THz}$ communication system with only 5-bit DACs and 5-bit ADCs can still achieve Tbps in the low-power regime. We have proven that the achievable rate for the single-user transmissions with finite-bit DACs and/or finite-bit ADCs is robust to the phase uncertainties in the large subarray antenna regime. Extensions to the imperfect CSI case (see e.g., [41] and [42]) will be interesting research topics and are left for future study.

\section{APPENDIX}

\section{A. Proof of Proposition 1}

Substituting (1) into (6), the effective channel between the subarray $k \in \mathcal{K}$ at the transmitter with analog beamforming angle $\left(\zeta_{t}, \theta_{t}\right)$ and a subarray at the user with analog beamforming angle $\left(\zeta_{r}, \theta_{r}\right)$ can be expressed as

$$
\begin{aligned}
\hat{h}_{k} & =\mathbf{a}_{r}^{H}\left(\zeta_{r}, \theta_{r}\right) \mathbf{F}_{s u b}(f, d) \mathbf{a}_{t}\left(\zeta_{t}, \theta_{t}\right) \\
& =\sqrt{M_{t} N_{t} M_{r} N_{r}}\left(\eta_{L}(f, d) \Omega_{t} \Omega_{r} \mathbf{a}_{r}^{H}\left(\zeta_{r}, \theta_{r}\right) \mathbf{a}_{r}\left(\psi_{L}^{r}, \phi_{L}^{r}\right)\right. \\
& \times \mathbf{a}_{t}^{H}\left(\psi_{L}^{t}, \phi_{L}^{t}\right) \mathbf{a}_{t}\left(\zeta_{t}, \theta_{t}\right)+\sum_{i=1}^{J} \eta_{i}(f, d) \Omega_{t} \Omega_{r} \\
& \left.\times \mathbf{a}_{r}^{H}\left(\zeta_{r}, \theta_{r}\right) \mathbf{a}_{r}\left(\psi_{i}^{r}, \phi_{i}^{r}\right) \mathbf{a}_{t}^{H}\left(\psi_{i}^{t}, \phi_{i}^{t}\right)\right) \mathbf{a}_{t}\left(\zeta_{t}, \theta_{t}\right) .
\end{aligned}
$$

Note that when $M_{t}, N_{t} \rightarrow \infty$, we have

$$
\begin{aligned}
& \lim _{M_{t}, N_{t} \rightarrow \infty}\left|\mathbf{a}_{t}^{H}\left(\psi_{L}^{t}, \phi_{L}^{t}\right) \mathbf{a}_{t}\left(\zeta_{t}, \theta_{t}\right)\right| \\
& =\lim _{M_{t}, N_{t} \rightarrow \infty} \mid \frac{1}{M_{t} N_{t}} e^{-j \frac{M_{t}-1}{2}\left[\frac{2 \pi a}{\lambda}\left(\beta_{L 1}\left(\zeta_{t}, \theta_{t}\right)\right)\right]} \\
& \times \frac{\sin \left[\frac{M_{t} \pi a}{\lambda}\left(\beta_{L 1}\left(\zeta_{t}, \theta_{t}\right)\right)\right]}{\sin \left(\frac{\pi a}{\lambda}\left(\beta_{L 1}\left(\zeta_{t}, \theta_{t}\right)\right)\right)} e^{-j \frac{N_{t}-1}{2}\left[\frac{2 \pi a}{\lambda}\left(\beta_{L 2}\left(\zeta_{t}, \theta_{t}\right)\right)\right]} \\
& \times \frac{\sin \left[\frac{N_{t} \pi a}{\lambda}\left(\beta_{L 2}\left(\zeta_{t}, \theta_{t}\right)\right)\right]}{\sin \left(\frac{\pi a}{\lambda}\left(\beta_{L 2}\left(\zeta_{t}, \theta_{t}\right)\right)\right)} \mid \\
& =\delta\left(\beta_{L 1}\left(\zeta_{t}, \theta_{t}\right)\right) \delta\left(\beta_{L 2}\left(\zeta_{t}, \theta_{t}\right)\right),
\end{aligned}
$$

where

$$
\begin{gathered}
\beta_{L 1}\left(\zeta_{t}, \theta_{t}\right)=\cos \zeta_{t} \sin \theta_{t}-\cos \psi_{L}^{t} \sin \phi_{L}^{t}, \\
\beta_{L 2}\left(\zeta_{t}, \theta_{t}\right)=\sin \zeta_{t} \sin \theta_{t}-\sin \psi_{L}^{t} \sin \phi_{L}^{t} .
\end{gathered}
$$

Similarly, we can show that

$\lim _{M_{r}, N_{r} \rightarrow \infty}\left|\mathbf{a}_{r}^{H}\left(\zeta_{t}, \theta_{t}\right) \mathbf{a}_{r}\left(\psi_{L}^{r}, \phi_{L}^{r}\right)\right|=\delta\left(\beta_{L 1}\left(\zeta_{r}, \theta_{r}\right)\right) \delta\left(\beta_{L 2}\left(\zeta_{r}, \theta_{r}\right)\right)$,

$\lim _{M_{t}, N_{t} \rightarrow \infty}\left|\mathbf{a}_{t}^{H}\left(\psi_{i}^{t}, \phi_{i}^{t}\right) \mathbf{a}_{t}\left(\zeta_{t}, \theta_{t}\right)\right|=\delta\left(\beta_{i 1}\left(\zeta_{t}, \theta_{t}\right)\right) \delta\left(\beta_{i 2}\left(\zeta_{t}, \theta_{t}\right)\right)$
$\lim _{M_{r}, N_{r} \rightarrow \infty}\left|\mathbf{a}_{r}^{H}\left(\zeta_{t}, \theta_{t}\right) \mathbf{a}_{r}\left(\psi_{i}^{r}, \phi_{i}^{r}\right)\right|=\delta\left(\beta_{i 1}\left(\zeta_{r}, \theta_{r}\right)\right) \delta\left(\beta_{i 2}\left(\zeta_{r}, \theta_{r}\right)\right)$,

where

$$
\begin{aligned}
\beta_{L 1}\left(\zeta_{r}, \theta_{r}\right) & =\cos \zeta_{r} \sin \theta_{r}-\cos \psi_{L}^{r} \sin \phi_{L}^{r}, \\
\beta_{L 2}\left(\zeta_{r}, \theta_{r}\right) & =\sin \zeta_{r} \sin \theta_{r}-\sin \psi_{L}^{r} \sin \phi_{L}^{r}, \\
\beta_{i 1}\left(\zeta_{t}, \theta_{t}\right) & =\cos \zeta_{t} \sin \theta_{t}-\cos \psi_{i}^{t} \sin \phi_{i}^{t}, \\
\beta_{i 2}\left(\zeta_{t}, \theta_{t}\right) & =\sin \zeta_{t} \sin \theta_{t}-\sin \psi_{i}^{t} \sin \phi_{i}^{t}, \\
\beta_{i}\left(\zeta_{r}, \theta_{r}\right) & =\cos \zeta_{r} \sin \theta_{r}-\cos \psi_{i}^{r} \sin \phi_{i}^{r}, \\
\beta_{i 2}\left(\zeta_{r}, \theta_{r}\right) & =\sin \zeta_{r} \sin \theta_{r}-\sin \psi_{i}^{r} \sin \phi_{i}^{r}
\end{aligned}
$$

Taking the limit of (69) as $M_{t}, N_{t}, M_{r}, N_{r}$ approach infinity and substituting (71), (74)-(76) into the resulting equation, we have

$$
\begin{aligned}
& \lim _{M_{t}, N_{t}, M_{r}, N_{r} \rightarrow \infty} \frac{\hat{h}_{k}}{\sqrt{M_{t} N_{t} M_{r} N_{r}} \Omega_{t} \Omega_{r}}= \\
& \eta_{L}\left(f_{u}^{w}, d_{u}\right) \delta\left(\beta_{L 1}\left(\zeta_{r}, \theta_{r}\right)\right) \delta\left(\beta_{L 2}\left(\zeta_{r}, \theta_{r}\right)\right) \\
& \times \delta\left(\beta_{L 1}\left(\zeta_{t}, \theta_{t}\right)\right) \delta\left(\beta_{L 2}\left(\zeta_{t}, \theta_{t}\right)\right)+\sum_{i=1}^{J} \eta_{i}\left(f_{u}^{w}, d_{u}\right) \\
& \times \delta\left(\beta_{i 1}\left(\zeta_{r}, \theta_{r}\right)\right) \delta\left(\beta_{i 2}\left(\zeta_{r}, \theta_{r}\right)\right) \delta\left(\beta_{i 1}\left(\zeta_{t}, \theta_{t}\right)\right) \delta\left(\beta_{i 2}\left(\zeta_{t}, \theta_{t}\right)\right) .
\end{aligned}
$$


Considering that the norm of the equivalent channel $\|\mathbf{h}\|^{2}=$ $\sum_{k}\left|\hat{h}_{k}\right|^{2}$ in (5), and noting that $\delta(x)=\left\{\begin{array}{ll}1, & x=0 \\ 0, & \text { else }\end{array}\right.$, we know that

$$
\begin{gathered}
\lim _{M_{t}, N_{t}, M_{r}, N_{r} \rightarrow \infty} \frac{\left|\hat{h}_{k}\right|^{2}}{M_{t} N_{t} M_{r} N_{r}\left(\Omega_{t} \Omega_{r}\right)^{2}}= \\
\begin{cases}\left|\eta_{L}\left(f_{u}^{w}, d_{u}\right)\right|^{2}, & \left(\zeta_{t}, \theta_{t}\right)=\left(\psi_{L}^{t}, \phi_{L}^{t}\right) \&\left(\zeta_{r}, \theta_{r}\right)=\left(\psi_{L}^{r}, \phi_{L}^{r}\right) \\
\left|\eta_{i}\left(f_{u}^{w}, d_{u}\right)\right|^{2}, & \left(\zeta_{t}, \theta_{t}\right)=\left(\psi_{i}^{t}, \phi_{i}^{t}\right) \&\left(\zeta_{r}, \theta_{r}\right)=\left(\psi_{i}^{r}, \phi_{i}^{r}\right) \\
0, & \text { else. }\end{cases}
\end{gathered}
$$

Obviously, (84), and hence (5) is maximized with the choice of $\left(\zeta_{t}, \theta_{t}\right)=\left(\psi_{L}^{t}, \phi_{L}^{t}\right)$ and $\left(\zeta_{r}, \theta_{r}\right)=\left(\psi_{L}^{r}, \phi_{L}^{r}\right)$ since $\left|\eta_{L}(f, d)\right|>\left|\eta_{i}(f, d)\right|, \forall i$.

\section{B. Proof of Proposition 2}

The idea of this proof is similar to [13, Section V]. We include the proof here for the sake of the reader. We can first obtain the radiation pattern of one antenna subarray associated with the transmit beamforming vector specified by $\left(\tilde{\psi}_{q}^{t}, \tilde{\phi}_{q}^{t}\right)$ for the AP as

$$
\begin{aligned}
& |A(\phi, \theta, f)|^{2}=A(\phi, \theta, f) A^{H}(\phi, \theta, f) \\
& =\frac{1}{M N} \sum_{m=0}^{M-1} \sum_{n=0}^{N-1} \sum_{m^{\prime}=0}^{M-1} \sum_{n^{\prime}=0}^{N-1} e^{j\left(\sigma_{m, n}(f)-\sigma_{m^{\prime}, n^{\prime}}(f)\right)} \\
& \times e^{j \frac{2 \pi a}{\lambda}\left(m-m^{\prime}\right) \beta_{1}+\left(n-n^{\prime}\right) \beta_{2}},
\end{aligned}
$$

where $\beta_{1}=\cos \tilde{\psi}_{q}^{t} \sin \tilde{\phi}_{q}^{t}-\cos \phi \sin \theta$ and $\beta_{2}=$ $\sin \tilde{\psi}_{q}^{t} \sin \tilde{\phi}_{q}^{t}-\sin \phi \sin \theta$. Since $\sigma_{m, n}(f)-\sigma_{m^{\prime}, n^{\prime}}$ is very small, we can apply the Taylor's expansion and have

$$
\begin{aligned}
e^{j\left(\sigma_{m, n}(f)-\sigma_{m^{\prime}, n^{\prime}}(f)\right)} \approx 1 & +j\left(\sigma_{m, n}(f)-\sigma_{m^{\prime}, n^{\prime}}(f)\right) \\
& -\frac{1}{2}\left(\sigma_{m, n}(f)-\sigma_{m^{\prime}, n^{\prime}}(f)\right)^{2} .
\end{aligned}
$$

Then the average radiation pattern of one antenna subarray at the transmitter can be written as

$\mathbb{E}\left[\left|A_{t}(\phi, \theta, f)\right|^{2}\right]=\left|A_{t}(\phi, \theta)\right|^{2}-\frac{\varepsilon_{t}^{2}(f)}{3}\left|A_{t}(\phi, \theta)\right|^{2}+\frac{\varepsilon_{t}^{2}(f)}{3}$

Similarly, the average radiation pattern at the receiver is

$\mathbb{E}\left[\left|A_{r}(\phi, \theta, f)\right|^{2}\right]=\left|A_{r}(\phi, \theta)\right|^{2}-\frac{\varepsilon_{r}^{2}(f)}{3}\left|A_{r}(\phi, \theta)\right|^{2}+\frac{\varepsilon_{r}^{2}(f)}{3}$.

Then, the equivalent channel in (30) becomes

$$
\begin{aligned}
& \tilde{\mathbf{h}}_{u}\left(f_{u}^{w}, d_{u}\right) \stackrel{M_{t}, N_{t}, M_{r}, N_{r} \rightarrow \infty}{\longrightarrow} \\
& \sqrt{M_{t} N_{t}-\left(M_{t} N_{t}-1\right) \frac{\varepsilon_{t}^{2}(f)}{3}+\frac{\varepsilon_{t}^{2}(f)}{3}} \\
& \times \sqrt{M_{r} N_{r}-\left(M_{r} N_{r}-1\right) \frac{\varepsilon_{r}^{2}(f)}{3}+\frac{\varepsilon_{r}^{2}(f)}{3}} \Omega_{t} \Omega_{r} \mathbf{t}_{u}\left(f_{u}^{w}, d_{u}\right), \\
& =\sqrt{M_{t} N_{t} M_{r} N_{r}} \sqrt{1-\left(1-\frac{1}{M_{t} N_{t}}\right) \frac{\varepsilon_{t}^{2}(f)}{3}+\frac{1}{M_{t} N_{t}} \frac{\varepsilon_{t}^{2}(f)}{3}} \\
& \times \sqrt{1-\left(1-\frac{1}{M_{r} N_{r}}\right) \frac{\varepsilon_{r}^{2}(f)}{3}+\frac{1}{M_{r} N_{r}} \frac{\varepsilon_{r}^{2}(f)}{3}} \Omega_{t} \Omega_{r} \mathbf{t}_{u}\left(f_{u}^{w}, d_{u}\right) .
\end{aligned}
$$

Compared with (30), we can see that the term $\mathbf{t}_{u}\left(f_{u}^{w}, d_{u}\right)$ remains unchanged, while the coefficients are decreased due to $\varepsilon_{t}(f)$ and $\varepsilon_{r}(f)$. Nevertheless, this coefficient change will not alter the derivations of the results in Theorem 1, Theorem 2 and Theorem 3. For example, in the proof of Theorem 1 , we will replace (30) with (89) in the first step. Then, the coefficient will be eliminated similar to $\Omega_{t} \Omega_{r}$ and the same equation as (38) can be derived. As a result, following the subsequent steps, we can get the same result in Theorem 1.

\section{REFERENCES}

[1] F. Boccardi, R.W. Heath, Jr., A. Lozano, T. Marzetta, and P. Popovski, "Five disruptive technology directions for 5G," IEEE Commun. Mag., vol. 52, no. 2, pp. 74 - 80, Feb. 2014.

[2] J. G. Andrews et al., "What Will 5G Be?" IEEE J.Sel. Areas Commun., vol. 32, no. 6, pp. 1065-1082, Jun. 2014.

[3] L. Lu, G. Y. Li, A. L. Swindlehurst, A. Ashikhmin, and R. Zhang, "An overview of massive MIMO: Benefits and challenges," IEEE J. Sel. Topics Sig. Proc., vol. 8, no. 5, pp. 742 - 758, May 2014.

[4] R. Piesiewicz et al., "Short-range ultra-broadband Terahertz communications: concepts and perspectives," IEEE Antennas Propag. Mag., vol. 49, no. 6 , pp. 24 - 39, Dec. 2007

[5] K. C. Huang and Z. Wang,"Terahertz terabit wireless communication," IEEE Microw. Mag., vol. 12, no. 4, pp. 108-116, Jun. 2011

[6] H. Song and T. Nagatsuma, "Present and future of Terahertz communications," IEEE Trans. Terahertz Science and Tech., vol. 1, no. 1, pp. 256-263, Sep. 2011.

[7] I. F. Akyildiz, J. M. Jornet, and C. Han, "Terahertz band: next frontier for wireless communicaions," Phys. Commun., vol. 12, no. 2, pp. 16 32, Sep. 2014.

[8] J.M.Jornet and I.F.Akyildiz, "Channel modeling and capacity analysis for electromagnetic wireless nanonetworks in the Terahertz band," IEEE Trans. Wireless Commun., vol. 10, no.10, pp.3211-3221, Oct. 2011.

[9] S. Priebe and T. Krner, "Stochastic modeling of THz indoor radio channels,"IEEE Trans. Wireless Commun., vol. 12, no. 9, pp. 4445C4455,Sep. 2013.

[10] A. F. Molisch, "Ultrawideband propagation channels-theory, measurement, and modeling," IEEE Trans. Vehi. Technol., vol. 54, no. 5, pp. 1528-1545, Sept. 2005.

[11] C. Han, A. O. Bicen, and I. F. Akyildiz, "Multi-ray channel modeling and wideband characterization for wireless communications in the Terahertz band," IEEE Trans. Wireless Commun., vol. 14, no. 5, pp. 2402 2412, May 2015.

[12] C. Han and I. F. Akyildiz, "Distance-aware bandwidth-adaptive resource allocation for wireless systems in the Terahertz band," IEEE Trans. Terahertz Science and Tech., vol. 6, no. 4, pp. 541 - 553, July 2016.

[13] C. Lin and G. Y. Li, "Indoor Terahertz communications: how many antenna arrays are needed?" IEEE Trans. Wireless Commun., vol. 14, no. 6, pp. 3097-3107, Jun. 2015.

[14] C. Lin and G Ye Li, "Adaptive beamforming with resource allocation for distance-aware multi-user indoor Terahertz communications," IEEE Trans. Wireless Comun., vol. 63, no. 8, pp. 2985-2995, Aug. 2015.

[15] C. Lin and G. Y. Li, "Terahertz communications: an array-of-subarrays solution," IEEE Commun. Mag., vol. 54, no. 12, pp. 124 - 131, Dec. 2016.

[16] A. Brighente, M. Cerutti, M. Nicoli, S. Tomasin and U. Spagnolini, "Estimation of Wireband Dynamic mmWave and THz Channels for 5G Systems and Beyond," IEEE Journal on Selected Areas in Communications(Early Access), doi:10.1109/JSAC.2020.3000889.

[17] S. Nie and I. F. Akyildiz, "Deep Kernel Learning-Based Channel Estimation in Ultra-Massive MIMO Communications at 0.06-10 THz," in IEEE GC Wkshps 2019, Waikoloa, HI, USA, pp. 1-6, Dec. 2019.

[18] I. Chafaa and M. Djeddou, "Improved channel estimation in mmWave communication system," in Seminar on DAT 2017, Algiers, Algeria, pp. 1-5, Mar. 2017.

[19] J. Wang, Z. Lan, C. Pyo, C. Sum, T. Baykas, J. Gao, A. Rahman, R. Funada, F. Kojima, H. Harada, S. Kato, Beamforming Codebook Design and Performance Evaluation for Millimeter-wave WPAN, IEICE Tech. Rep., vol. 108, no. 445, RCS2008-286, pp. 437-442, March 2009.

[20] J. Wang et al., "Beam codebook based beamforming protocol for multiGbps millimeter-wave WPAN systems," IEEE J. Sel. Areas Commun., vol. 27, no. 8, pp. 1390C1399, Oct. 2009.

[21] S. Jacobsson, G. Durisi, M. Coldrey, T. Goldstein, and C. Studer, "Quantized precoding for massive MU-MIMO," IEEE Trans. Commun., vol. 65 , no. 11 , pp. 4670 - 4684, Nov. 2017. 
[22] S. Jacobsson, G. Durisi, M. Coldrey and C. Studer, "Linear Precoding With Low-Resolution DACs for Massive MU-MIMO-OFDM Downlink," IEEE Trans. Wireless Commun., vol. 18, no. 3, pp. 1595-1609, March 2019.

[23] A. K.Saxena, I. Fijalkow. A. L. Swindlehurst, "Analysis of one-Bit quantized precoding for the multiuser massive MIMO downlink," IEEE Trans. Signal processing., vol. 65, no. 17, pp.4624 - 4634, Sep. 2017.

[24] H. Jedda, A. Mezghani, J. A. Nossek, and A. L. Swindlehurst, "Massive MIMO downlink 1-bit precoding with linear programming for PSK signaling," in IEEE SPAWC 2017, Hokkaido, Japan, July 2017.

[25] L. T. N. Landau and R. C. D. Lamare, "Branch-and-Bound Precoding for Multiuser MIMO Systems With 1-Bit Quantization," IEEE Trans. Wireless Commun. Lett, vol. 6, no. 6, pp. 770-773, Dec. 2017.

[26] C. Wang, C. Wen, S. Jin and S. Tsai, "Finite-Alphabet precoding for massive MU-MIMO with low-resolution DACs," IEEE Trans. Wireless Commun., vol. 17, no. 7, pp. 4706-4720, Jul. 2018.

[27] L. N. Ribeiro, S. Schwarz, M. Rupp, and A. L.F. de Almeda, "Energy efficiency of mmWave massive MIMO precoding with low-resolution DACs," IEEE J. Sel. Topics in Sig. Proc., vol. 12 no. 2, pp. 298-312, May 2018.

[28] A. Li, C. Masouros, F. Liu and A. Swindlehurst, "Massive MIMO 1Bit DAC transmission: a low-complexity symbol scaling approach," IEEE Trans. Wireless Commun., vol.17, no. 11, pp, 7559-7575, Nov. 2018.

[29] O. Orhan, E. Erkip, and S. Rangan, "Low power analog-to-digital conversion in millimeter wave systems: impact of resolution and bandwidth on performance,' in 2015 ITA, San Diego, CA, Feb. 2015.

[30] B. Li, N. Liang, and W. Zhang, "On transmission model for massive MIMO under low-resolution output quantization," in Proc. IEEE Veh. Technol. Conf. Spring(VTC-Spring), Sydney, NSW, Australia, Jun. 2017.

[31] M. M. Molu, P. Xiao, M. Khalily, K. Cumanan, L, Zhang, and R Tafazolli. " Low-complexity and robust hybrid beamforming design for multi-antenna communication systems." IEEE Trans. Wireless Commun., vol. 17, no. 3, pp. 1445-1459, Mar. 2018.

[32] J. Yuan, S. Jin, C. Wen, K, Wong, "The distributed MIMO scenario: can ideal ADCs be replaced by low-resolution ADCs?" IEEE Wireless Comun. Lett, vol. 6, no. 4, Aug. 2017.

[33] S. Jacobsson, G. Durisi, M. Coldrey, T. Goldstein, C. Studer,"Throughput analysis of massive MIMO uplink with low-resolution ADCs," IEEE Trans. Wireless Commun., vol. 16, no. 6, pp. 4038 - 4051, Jun. 2017.

[34] A. Mezghani and J. A. Nossek, "Capacity lower bound of MIMO channels with output quantization and correlated noise," in Proc. IEEE. ISIT, Cambridge, MA, USA, pp. 1-5. Jul. 2012.

[35] J. Mo and R. Heath, "Capacity analysis of one-bit quantized MIMO systems with transmitter channel state information," IEEE Trans. Signal processing., vol. 63, no. 20, pp.5498 - 5512, Oct. 2015.

[36] L. N. Landau, M. Dörpinghaus, R. C. de lamare and G. Fettweis, "Achievable rate with 1-bit quantization and oversampling using continuous phase modulation-based sequences," IEEE Trans. Wireless Commun., vol. 17, no. 10, pp. 7080-7095, Jun. 2018.

[37] Q. Hou, R. Wang, E. Liu and D. Yan, "Hybrid precoding design for MIMO system with one-bit ADC receivers," IEEE Access, vol.6, pp. 48478-48488, Sep. 2018.

[38] C. Molln, J, Choi, E. G. Larsson, and R. W. Heath, "Uplink performance of wideband massive MIMO with one-bit ADCs," IEEE Trans. Wireless Commun., vol. 16, no. 1, pp. 87-100, Jun. 2017.

[39] J. Mo and R. W. Heath "Limited feedback in single and multi-user MIMO systems with finite-bit ADCs," IEEE Trans. Wireless Commun., vol. 17, no. 5, pp. 3284-3297, May. 2018.

[40] M. S. Stein, S. Bar, J. A. Nossek and J. Tabrikian "Performance analysis for channel estimation with 1-bit ADC and unknown quantization threshold," IEEE Trans. Signal processing., vol. 66, no. 10, pp. 25572571, May. 2018.

[41] P. Dong, H. Zhang, W. Xu and X. You, "Efficient Low-Resolution ADC Relaying for Multiuser Massive MIMO System," IEEE Trans. Vehi. Technol., vol. 66, no. 12, pp. 11039-11056, Dec. 2017.

[42] P. Dong, H. Zhang, Q. Wu and G. Y. Li, "Spatially Correlated Massive MIMO Relay Systems With Low-Resolution ADCs," IEEE Trans. Vehi. Technol., vol. 69, no. 6, pp. 6541-6553, June 2020.

[43] J. Max, "Quantizing for minimum distortion," IRE Trans. Inf. Theory, vol. 6, no. 1, pp. 7-12, March 1960.

[44] J. Xu, W. Xu, F. Shi, and H. Zhang, "User loading in downlink multiuser massive MIMO with 1-bit DAC and quantized receiver," in IEEE VTC 2017-Fall, Toronto, CA, Sep. 2017.

[45] J. Xu, W. Xu, and F. Gong, "On performance of quantized transceiver in multiuser massive MIMO downlinks," IEEE Wireless Comun. Lett, vol. 6 , no. 5 , Oct. 2017.
[46] C. Kong, A. Mezghani, C. Zhong, A. Swindlehurst and Z. Zhang, "Multipair massive MIMO relaying systems with one-bit ADCs and DACs," IEEE Trans. Signal processing., vol. 66, no. 11, pp.2984 - 2997, Jun. 2018.

[47] J. Zhang, L. Dai, Z. He, B. Ai and O. Dobre, "Mixed-ADC/DAC multipair massive MIMO relaying systems: performance analysis and power optimization," IEEE Trans. Commun., vol. 67, no. 1, pp. 140-153, Jan. 2019.

[48] C. Kong, A. Mezghani, C. Zhong, A. Swindlehurst and Z. Zhang, "Nonlinear precoding for multipair relay networks with one-bit ADCs and DACs," IEEE Signal processing. Lett, vol. 25, no. 2, pp.303 - 307, Feb. 2018.

[49] A. B. Üçüncü and A. O. Yılmaz, "Oversampling in one-bit quantized massive MIMO systems and performance analysis," IEEE Trans. Wireless Commun., vol. 17, no. 12, pp. 7952-7964, Dec. 2018.

[50] G. Jacovitti and A. Neri, "Estimation of the autocorrelation function of complex Gaussian stationary processes by amplitude clipped signals," IEEE Trans. Inf. Theory, vol. 40, no. 1, pp. 239 - 245, Jan. 1994.

[51] J. J. Bussgang, "Crosscorrelation functions of amplitude-distorted Gaussian signals," Res. Lab. Elec., Cambridge, MA, Tech. Rep. 216, Mar. 1952.

[52] P. Y. Chen, C. Argyropoulos, and A. Alù,"Terahertz antenna phase shifters using integrally-gated graphene transmission-lines," IEEE Trans. Anten. Propag., vol. 61, no. 4, pp. 1528C1537, Apr. 2013.

[53] J. Wang et al., "Beam codebook based beamforming protocol for multiGbps millimeter-wave WPAN systems," IEEE J. Sel. Areas Commun., vol. 27 , no. 8, pp. 1390C1399, Oct. 2009.

[54] X. Luo, "Multiuser massive MIMO performance with calibration errors," IEEE Trans. Wireless Commun., vol. 15, no. 7, pp. 4521-4534, July 2016.

[55] X. Jiang, A. Decurninge, K. Gopala, F. Kaltenberger, M. Guillaud, D. Slock, and L. Deneire, "A framework for over-the-air reciprocity calibration for TDD massive mimo systems," IEEE Trans. Wireless Commun., vol. 17, no. 9, pp. 5975-5990, Sep. 2018.

[56] M. Pesavento, A. B. Gershman, and K. M. Wong, "Direction finding in partly calibrated sensor arrays composed of multiple subarrays," IEEE Trans. Sig. Proc., vol. 50, no. 9, pp. 2103-2115, Sep. 2002.

[57] S. A. Elkader, A. B. Gershman, and K. M. Wong, "Rank reduction direction-of-arrival estimators with an improved robustness against subarray orientation errors," IEEE Trans. Sig. Proc., vol. 54, no. 5, pp. 1951 1955, May 2006.

[58] L. Lei, J. P. Lie, A. B. Gershman, and C. M. S. See, "Robust adaptive beamforming in partly calibrated sparse sensor arrays," IEEE Trans. Sig. Proc., vol. 58, no. 3, pp. 1661-1667, Mar. 2010.

[59] D. M. Arnold, H.-A. Loeliger, P. O. Vontobel, A. Kavcic, and W. Zeng, "Simulation-based computation of information rates for channels with memory," IEEE Trans. Inf. Theory, vol. 52, no. 8, pp. 3498C3508, Aug. 2006.

[60] M. Yu, A. Tang, X. Wang, and C. Han, "Joint scheduling and power allocation for 6G terahertz mesh networks," in 2020 International Conference on Computing, Networking and Communications (ICNC).

[61] D. Li, D. Qiao, L. Zhang, "Achievable rate of indoor THz communication systems with finite-bit ADCs" in IEEE WCSP 2018, Hangzhou, China, Oct. 2018.

[62] D. Li, D. Qiao, L. Zhang, and G. Y. Li, "Performance analysis of indoor $\mathrm{THz}$ communications with one-bit precoding" in IEEE GLOCOM 2018, Abu Dhabi, United Arab Emirates, Dec. 2018.

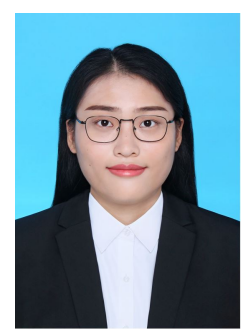

Yujiao Zhang received the B.S. degree from East China Normal University in 2019. She is currently pursuing the Ph.D degree with the School of Communication and Electronic Engineering, East China Normal University, Shanghai, China. Her current research interest includes low-resolution DACs and energy efficiency in Terahertz Communications. 


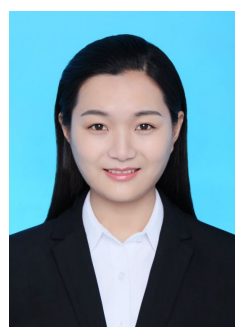

Dan $\mathbf{L i}$ is currently an Engineer at Huawei Technologies Inc. Previous, she received the M.S. degree from the East China Normal University in 2019.

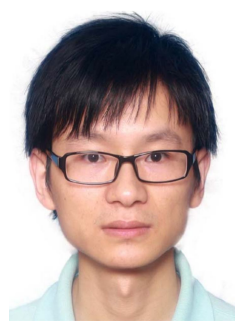

Deli Qiao received the B.E. degree in electrical engineering from Harbin Institute of Technology in 2007, and the Ph.D. degree in electrical engineering from the University of Nebraska Lincoln in 2012. Currently, he is a Professor at the East China Normal University, China. From 2012 to 2014, he worked in Huawei Technologies Inc. as a research engineer, focusing on $5 \mathrm{G}$ techniques especially massive MIMO. His research interests are in the general areas of wireless communications, information theory, and signal processing. He has been an Editor of CHINA COMMUNICATIONS since 2016. He was a recipient of the Technical Committee on Green Communications and Computing (TCGCC) Best Journal Paper Award in 2017. He received the Maude Hammond Fling Fellowship from the University of Nebraska Lincoln in 2011.

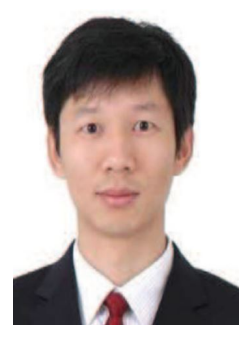

Lei Zhang is a Senior Lecturer at the University of Glasgow. He received his Ph.D. degree from the University of Sheffield, U.K. He worked as a research fellow in the 5G Innovation Centre (5GIC), University of Surrey, U.K., and a research engineer in Huawei.

$\mathrm{He}$ has more than 10 years academia and industry combined research experience on $3 \mathrm{G} / 4 \mathrm{G} / 5 \mathrm{G}$ wireless communications and its applications to IoT, Blockchain and automation. He is the PI or a Co-I of research projects worth $£ 2.6 \mathrm{~m}+$, funded by UKRI, DCMS, Scottish government and industry. His 19 international patents are granted/filed in 30+ countries including USA/UK/EU/China/Japan etc. He has $100+$ peer-reviewed publications, including 2 books, 50+ journal papers $(45+$ in IEEE journals, 20+ in IEEE Transactions, 17 as the first author), 50+ conference papers and book chapters.

Dr Zhang is an associate editor of IEEE Internet of Things (IoT) Journal, IEEE Wireless Communications Letters and Digital Communications and Networks. He is a Senior Member of IEEE. He received the IEEE ICC TAOS Best Paper Award 2019. Dr. Lei Zhang was Technical Program Chair for 5th International Conference on UCET 2020, Publication and Registration Chair of IEEE SAM 2018, Co-chair of Cyber-C 2019 Blockchain workshop. 